\title{
Article \\ Genetic Comparison of ESBL-Producing Escherichia coli from Workers and Pigs at Vietnamese Pig Farms
}

\author{
Duong Thi Quy Truong ${ }^{1}$, Yaovi Mahuton Gildas Hounmanou ${ }^{2, *} \mathbb{D}$, Son Thi Thanh Dang ${ }^{1}$, \\ John Elmerdahl Olsen ${ }^{2}{ }^{\mathbb{D}}$, Giang Thi Huong Truong ${ }^{1}$, Nhat Thi Tran ${ }^{1} \mathbb{D}$, Flemming Scheutz ${ }^{3}$ \\ and Anders Dalsgaard ${ }^{2, *(1)}$
}

1 National Institute of Veterinary Research, Hanoi 10000, Vietnam; truongduong2603@gmail.com (D.T.Q.T.); chienson2006@yahoo.com (S.T.T.D.); tgiang.vty83@yahoo.com (G.T.H.T.); trannhatbn1991@gmail.com (N.T.T.)

2 Department of Veterinary and Animal Sciences, University of Copenhagen, 1870 Frederiksberg C, Denmark; jeo@sund.ku.dk

3 Department of Bacteria, Parasites and Fungi, Statens Serum Institute, 2300 Copenhagen S, Denmark; fsc@ssi.dk

* Correspondence: gil@sund.ku.dk (Y.M.G.H.); adal@sund.ku.dk (A.D.)

Citation: Truong, D.T.Q.;

Hounmanou, Y.M.G.; Dang, S.T.T.; Olsen, J.E.; Truong, G.T.H.; Tran, N.T.;

Scheutz, F; Dalsgaard, A. Genetic Comparison of ESBL-Producing

Escherichia coli from Workers and Pigs at Vietnamese Pig Farms. Antibiotics 2021, 10, 1165. https://doi.org/ 10.3390/antibiotics10101165

Academic Editors:

Massimiliano Orsini and

Carmen Losasso

Received: 20 August 2021

Accepted: 22 September 2021

Published: 25 September 2021

Publisher's Note: MDPI stays neutral with regard to jurisdictional claims in published maps and institutional affiliations.

Copyright: (C) 2021 by the authors Licensee MDPI, Basel, Switzerland. This article is an open access article distributed under the terms and conditions of the Creative Commons Attribution (CC BY) license (https:/ / creativecommons.org/licenses/by/ $4.0 /)$.

\begin{abstract}
We analyzed and compared genomes of Extended Spectrum Beta-Lactamase (ESBL)producing Escherichia coli from pigs and pig farm workers at 116 farms in Vietnam. Analyses revealed the presence of $b l a_{\mathrm{CTX}-\mathrm{M}-55}, b l a_{\mathrm{CTX}-\mathrm{M}-27}, b l a_{\mathrm{CTX}-\mathrm{M}-15}, b l a_{\mathrm{CTX}-\mathrm{M}-14}, b l a_{\mathrm{CTX}-\mathrm{M}-3}, b l a_{\mathrm{CTX}-\mathrm{M}-65}$, $b l a_{C T X-M-24}, b l a_{D H A-1}$, and $b l a_{C M Y 2}$ in both hosts. Most strains from pigs contained quinolones ( $q n r$ ) and colistin resistance genes ( $m c r-1$ and $m c r-3)$. Isolates predominantly harbored more than one plasmid replicon and some harbored plasmid replicons on the same contigs as the ESBL genes. Five strains from farm workers of ST38 (2), ST69 (1), and ST1722 (2) were classified as either uropathogenic E. coli $\left(\mathrm{UPEC}_{\mathrm{HM}}\right)$ /extraintestinal pathogenic E. coli $\left(\mathrm{ExPEC}_{\mathrm{JJ}}\right)$ or $\mathrm{UPEC}_{\mathrm{HM}}$, and the remaining were genetically distinct commensals. A high heterogeneity was found among the ESBL-producing E. coli from pigs and workers, with most isolates belonging to unrelated phylogroups, serogroups, and sequence types with $>4046$ Single-Nucleotide Polymorphisms-(SNPs). In comparing the genomes of pig isolates to those from humans, it appeared that ESBL-producing E. coli in workers did not predominantly originate from pigs but were rather host-specific. Nevertheless, the occurrence of ESBL-producing E. coli carrying plasmid-mediated colistin and quinolone resistance genes in pigs could represent a potential source for horizontal transmission to humans through food rather than direct contact.
\end{abstract}

Keywords: ESBL; genomics; commensal E. coli; one health

\section{Introduction}

The World Health Organization (WHO) regards antimicrobial resistance (AMR) as one of the most important threats to public health because of the diminished effectiveness of antimicrobial treatment [1]. Studies have shown that extensive use of antimicrobials in livestock production affects AMR in humans [2,3] and both resistant bacteria in livestock and food should therefore be monitored.

Pork is the main meat consumed by people in Vietnam and, on average, each member of the country's 95 million people consumes $29 \mathrm{~kg}$ pork per year [4]. The pig production is the fifth largest in the world with 2386 metric tons of pork meat [5]. Production is mainly carried out on small-scale farms with a low level of biosecurity and hygiene, as well with frequent use of antimicrobials for disease prevention and treatment, and as growth promoters in commercial feed [6]. Thus, it has been estimated that, on average, 287 to $564 \mathrm{mg}$ active antimicrobials are used per kilogram of live pig produced in Vietnam [6,7].

Antimicrobials of critical importance in human medicine such as colistin [8] are used for prophylaxis and treatment of pigs in Vietnam [9]. Increasing levels of $\beta$-lactams, 
including cephalosporin, and colistin resistance have been reported as, e.g., 100\% of E. coli isolated from pigs were resistant to ampicillin and $40 \%$ of isolates were resistant to colistin $[9,10]$. Dang et al. [10] found that $89 \%$ of E. coli from pigs in small and medium-scale farms in Northern Vietnam were resistant to cefotaxime, a third generation cephalosporin. A high level of resistance to gentamycin, ciprofloxacin, and third generation cephalosporins was also found on chicken farms in Southern Vietnam [11].

High levels of bacterial antimicrobial resistance is not only found in livestock but also in the food and people in Vietnam [12]. It is increasingly suggested that production animals are important sources of the antimicrobial resistant bacteria in farm workers. While it is well-documented that resistant bacteria can be transmitted through the food chain, there is a paucity of evidence that direct contact is a main route of transfer for enteric resistant bacteria, including ESBL-producing E. coli, from animals to farmers [13-16]. The present study aims to determine and compare the genetic characteristics of ESBL-producing E. coli from pigs and pig farm workers. To address this aim, we used whole-genome sequencing (WGS) to analyze ESBL-producing E. coli isolated from fecal samples of pigs and pig farmers in Bac Ninh Province in Northern Vietnam.

\section{Results}

\subsection{Antimicrobial Resistance in E. coli Isolated from Pigs and Farm Workers}

E. coli was isolated from MacConkey agar without cefotaxime from all pig fecal samples (116), while only 94 fecal samples from farm workers yielded E. coli. The susceptibility to antimicrobials was determined for one randomly picked and confirmed E. coli isolate per fecal sample (Table 1). The results showed that the resistance levels in pigs were slightly higher than in farm workers. For instance, resistance to ampicillin (AMP), sulfonamide (SUL), trimethoprim (TMP), and streptomycin (STR) was present in 86\% (100/116), $82 \%(96 / 116), 72 \%(84 / 116)$, and $56 \%(65 / 116)$ of the pig isolates, respectively, compared to $72 \%(68 / 94), 67 \%(63 / 94), 65 \%(61 / 94)$, and $42 \%$ (40/94) of the isolates from farm workers. There was a significant difference of resistance levels to AMP $(p=0.02)$ and SUL $(p=0.01)$ in isolates from pigs and farm workers. Resistance to colistin (COL) in the pig isolates was 38\% (44/116), which was much higher $(p=0.003)$ than in the human isolates $(15 \%$; 14/94). Resistance to cephalosporin drugs, including ceftiofur (EFT), ceftriaxone (CRO), and cefoxitin (FOX), was, however, higher in the isolates from the workers than in the pig isolates (Table 1). Three isolates from pigs and seven isolates from workers were fully susceptible to all tested antimicrobials. Sixty-two out of 94 human isolates $(66 \%)$ were classified as multi-drug resistant (MDR), while 96 isolates were MDR among the 116 pig isolates (82\%). A total of 55 different AMR profiles were observed among the 116 pig E. coli isolates, while 50 profiles were found among the isolates from the farm workers (Table S1). Twenty-two profiles were shared between pig and human isolates, accounting for $59 \%$ of the total isolates.

Table 1. Prevalence of antimicrobial resistance in E. coli isolated from pigs and pig farm workers.

\begin{tabular}{|c|c|c|c|}
\hline Antimicrobial & Farm Worker Feces $(\mathrm{N}=94)$ & Pig Manure (N = 116) & $p$-Value \\
\hline & $\begin{array}{c}\text { Number of Resistant Isolates } \\
n(\%)\end{array}$ & $\begin{array}{c}\text { Number of Resistant Isolates } n \\
(\%)\end{array}$ & \\
\hline Ampicillin (AMP) & $68(72.3)$ & $100(86.2)$ & 0.02 \\
\hline Gentamycin (GEN) & 11 (11.7) & $34(29.3)$ & 0.003 \\
\hline Trimethoprim (TMP) & $61(64.9)$ & $84(72.4)$ & 0.30 \\
\hline Tetracycline (TET) & $68(72.3)$ & $98(84.5)$ & 0.04 \\
\hline Streptomycin (STR) & $40(42.6)$ & $65(56.0)$ & 0.07 \\
\hline Nalidixic acid (NAL) & $25(26.6)$ & $32(27.6)$ & 0.99 \\
\hline Sulphonamide (SUL) & $63(67.0)$ & $96(82.8)$ & 0.01 \\
\hline Ciprofloxacin (CIP) & 11 (11.7) & 17 (14.7) & 0.67 \\
\hline Ceftriaxone (CRO) & $13(13.8)$ & $1(0.9)$ & 0.0005 \\
\hline Cefoxitin (FOX) & $4(4.3)$ & $0(0.0)$ & \\
\hline $\begin{array}{l}\text { Amoxicillin-clavulanic acid } \\
\text { (AMC) }\end{array}$ & $4(4.3)$ & $3(2.6)$ & \\
\hline Ceftiofur (EFT) & $13(13.8)$ & $1(0.9)$ & 0.0005 \\
\hline Colistin (COL) & $14(14.9)$ & $44(37.9)$ & 0.003 \\
\hline
\end{tabular}




\subsection{Occurrence of ESBL-Producing E. coli in Pigs and Pig Farm Workers}

Fifty-eight of the 94 (62\%) human samples and 87/116 (75\%) of the pig samples yielded presumptive E. coli colonies on MacConkey agar containing $2 \mathrm{mg} / \mathrm{L}$ cefotaxime. One isolate from each sample confirmed as $E$. coli in the phenotypic testing was picked and tested for the ESBL phenotype. A total of 43/58 (74\%) isolates from workers and 78/87 $(90 \%)$ of the pig isolates were confirmed as ESBL-producing E. coli by the double-disk synergy test. These ESBL strains were all subjected to further antimicrobial resistance testing, in which all the isolates were found resistant to AMP, CRO, and EFT (Figure 1). Co-occurrence of resistance to colistin was observed in $51 \%$ of the pig isolates $(40 / 78)$ and $21 \%$ of the human isolates $(9 / 43)$. Forty-nine resistance profiles were found among the 121 ESBL-producing E. coli (Table S2), of which 11 profiles were shared between the human and pig isolates.

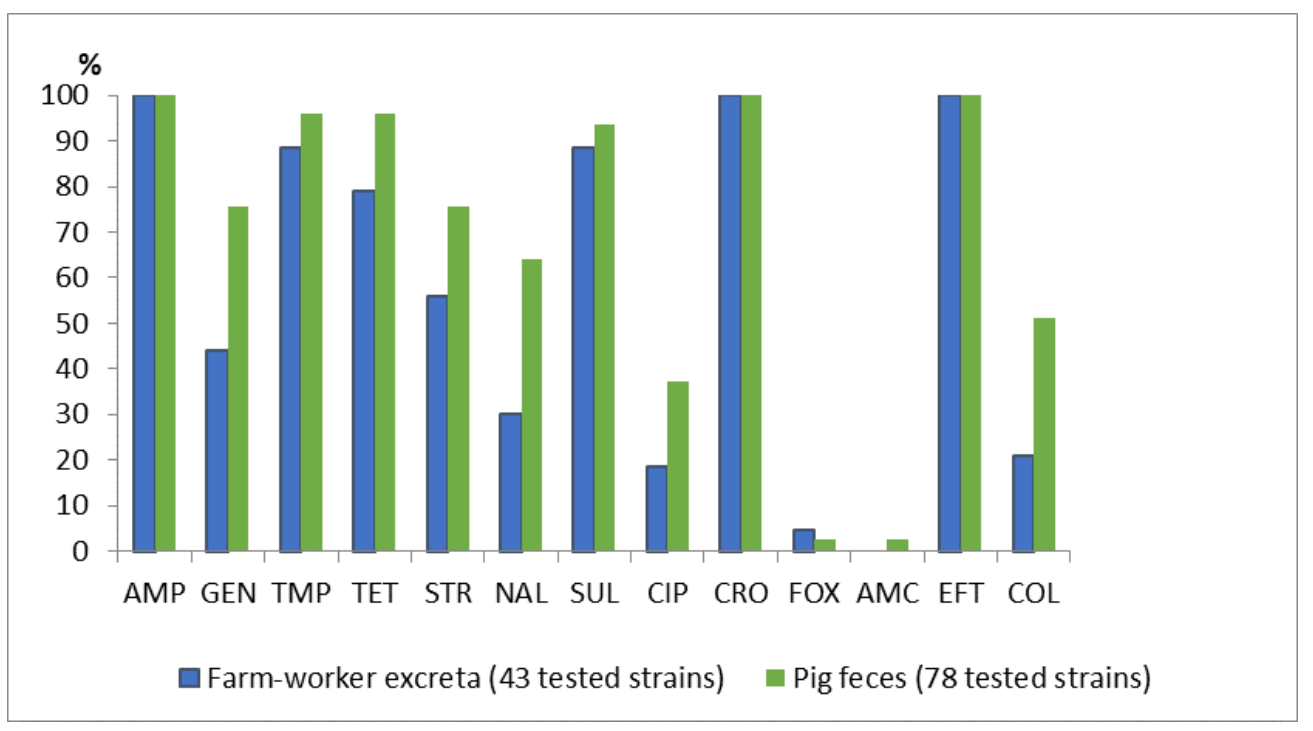

Figure 1. Antimicrobial resistance of ESBL-producing E. coli from pigs and pig farm workers. Abbreviations: AMP, Ampicillin; GEN, Gentamycin; TMP, Trimethoprim; TET, Tetracycline; STR, Streptomycin; NAL, Nalidixic acid; SUL, Sulphonamide; CIP, Ciprofloxacin; CRO, Ceftriaxone; FOX, Cefoxitin; AMC, Amoxicillin-clavulanic acid; EFT, Ceftiofur; and COL, Colistin.

\subsection{Antimicrobial Resistance Genes in ESBL-Producing E. coli \\ 2.3.1. ESBL Genes}

Seventy-four ESBL isolates were selected for WGS analysis (see Materials and Methods for selection strategy). The ESBL genes detected in the 43 pig isolates were $b a_{\mathrm{CTX}-\mathrm{M}-55}$ $(22 / 43)$, bla $_{\mathrm{CTX}-\mathrm{M}-14}(10 / 43)$, bla $_{\mathrm{CTX}-\mathrm{M}-27}(5 / 43)$, bla $_{\mathrm{OXA}-10}(4 / 43)$, bla $_{\mathrm{CTX}-15}(2 / 43)$, and $b a_{\mathrm{CTX}-\mathrm{M}-65}(1 / 43)$ (Figure 2). Two of the isolates harboring $b l a_{\text {OXA-10 }}$ also carried $b a_{\mathrm{CTX}-\mathrm{M}-55}$, while the other two isolates contained bla $a_{\mathrm{CTX}-\mathrm{M}-27}$. One isolate (Ec-67 from farm E-04) harboring $b l a_{\mathrm{CTX}-\mathrm{M}-14}$ co-carried an $A m p \mathrm{C}$ gene $\left(b l a_{\mathrm{DHA}-1}\right)$. The ESBL genes detected in the human isolates included bla $a_{\mathrm{CTX}-\mathrm{M}-27}(11 / 31)$, bla ${ }_{\mathrm{CTX}-\mathrm{M}-55}(10 / 31)$, bla $_{\mathrm{CTX}-15}(4 / 31)$, and $b l a_{\mathrm{CTX}-\mathrm{M}-14}(3 / 31)$. The $b l a_{\mathrm{CARB}-2}$ gene was found in two isolates also carrying $b l a_{\mathrm{CTX}-\mathrm{M}-55}$. The $b l a_{\mathrm{CTX}-\mathrm{M}-3}, b l a_{\mathrm{CTX}-\mathrm{M}-24}$, and $b l a_{\mathrm{CTX}-\mathrm{M}-65}$ genes were found in three human isolates. Two isolates from farm workers carrying bla $_{\mathrm{CTX}-\mathrm{M}-27}$ were also shown to harbor $A m p \mathrm{C}$ genes; one isolate carried $b l a_{\mathrm{DHA}-1}$ and another isolate had $b l a_{\mathrm{CMY}-2}$. Two isolates from workers harbored two bla genes, i.e., bla $a_{\mathrm{CTX}-\mathrm{M}-55}$ and $b l a_{\mathrm{CARB}-2}$ (Figure 2). Overall, bla $a_{\mathrm{CTX}-\mathrm{M}}$ genes were the most dominant of ESBL genes in all the samples from pigs and pig farm workers. Two AmpC genes, namely $b l a_{\mathrm{DHA}-1}$ and $b l a_{\mathrm{CMY}-2}$, were always co-occurring with other bla genes. 

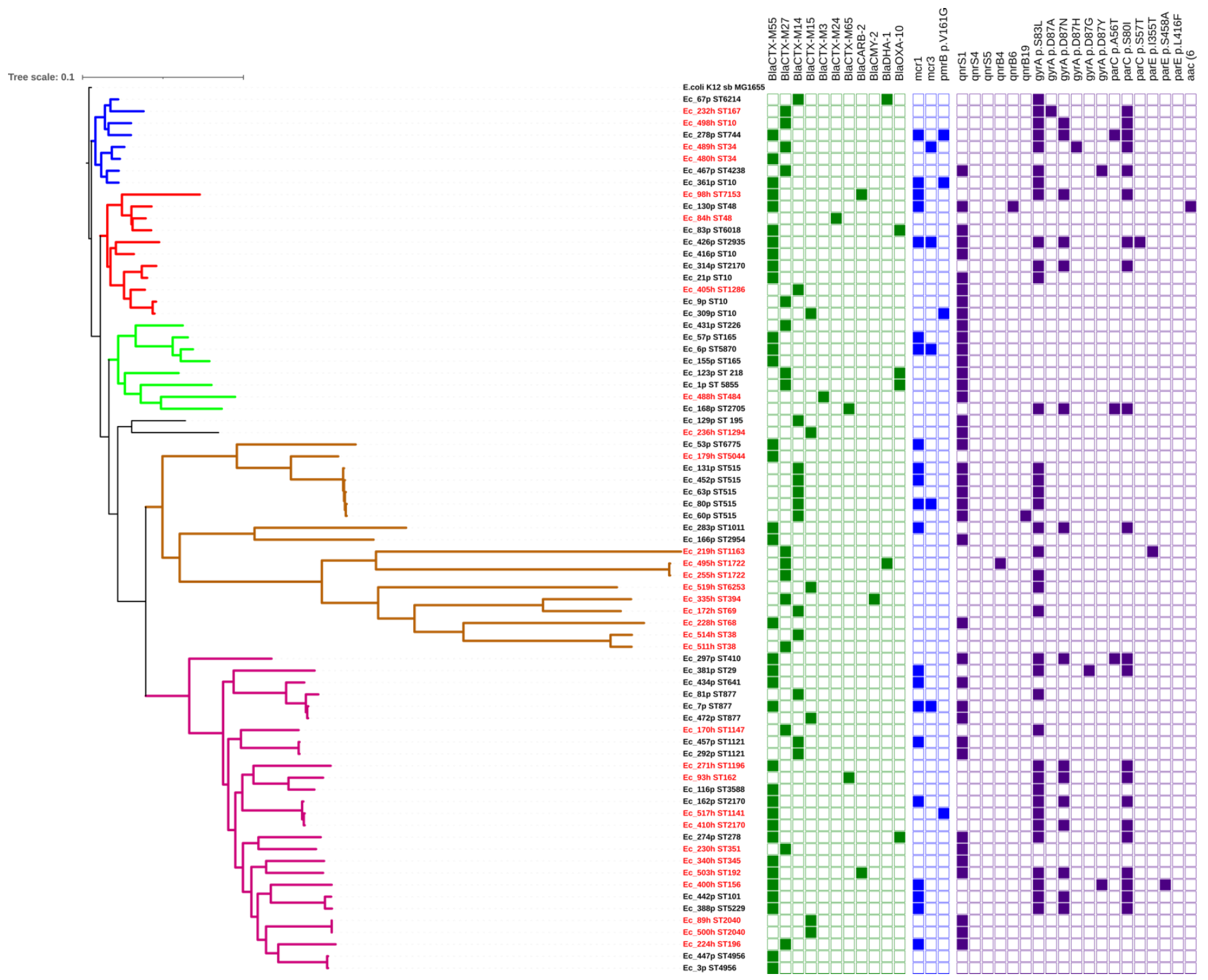

Figure 2. Phylogeny of ESBL-producing E. coli from pigs and pig farm workers. The isolates from workers are shown with red font node labels and the pig isolates are in black. The squares to the right indicate gene presence (full square) and absence (empty square). Note: green, ESBL genes; blue, plasmid-mediated colistin resistance genes and mutations; and purple, quinolone resistance (plasmid-mediated genes and point mutations). The color distinctions of the tree branches distinguish six main clusters in which the 74 isolates were regrouped.

\subsubsection{Colistin Resistance Genotypes}

Eighteen of the 43 ESBL-producing $E$. coli isolates from pigs (four with $b l a_{\mathrm{CTX}-\mathrm{M}-14}$ and 14 with $\left.b l a_{\mathrm{CTX}-\mathrm{M}-55}\right)$ carried colistin resistance genes. All these 18 isolates carried the $m c r-1$ gene, of which four isolates also contained $m c r-3$. Moreover, three of those having only $m c r-1$ revealed a mutation on the $p m r$ B gene at position p.V161G (Valine- > Glycin), encoding colistin resistance (Figure 2). However, one isolate (Ec_309) had only the $p m r$ B mutation and was susceptible to colistin in the phenotypic test. Among the isolates from farm workers, six isolates were found to be colistin-resistant in the MIC test. Three isolates (two isolates harboring $b l a_{\mathrm{CTX}} \mathrm{M}-55$ and one isolate harboring $b l a_{\mathrm{CTX}-\mathrm{M}-27}$ ) carried the $m c r-1$ gene, while one isolate (Ec489 with $b l_{\text {CTX-M-27 }}$ ) carried only the $m c r-3$ gene. The two other isolates (Ec_219-Farm A10 and Ec_255-farm E05) showing colistin-resistant phenotypes did not carry a resistance gene or known mutation that supports such resistance. Overall, the presence of $m c r-1$ and $m c r-3$ together with mutations in the $p m r B$ gene were 
the main genotypes associated with colistin resistance in the analyzed ESBL-producing E. coli genomes and were encountered more in the pig rather than human isolates.

\subsubsection{Quinolone Resistance}

The genes qnrB4, qnrB6, qnrB19, qnrS1, qnrS4, and qnrS5, belonging to the plasmidmediated quinolone resistance category (PMQR) were found in one or more of the 74 ESBLproducing E. coli. The $q n r S 1$ gene was the most commonly found and was detected in 10 human isolates and 29 pig isolates. Some isolates carried more than one PMQR gene, e.g., the pig isolate Ec60-farm E01 co-carried qnrS1 and qnrB19 genes, and the pig isolate Ec_130-farm B09 carried qnrB6 and qnrS1 (Figure 2). Mutations in the quinolone resistancedetermining region (QRDR) of topoisomerase genes were found in 51\% (22/43) of the pig isolates and in 17 of the human isolates, resulting in amino acid substitutions in the gyr $A$ as well as in parC and parE (Table 2).

Table 2. Mutations in the quinolone resistance-determining region (QRDR) of ESSBL-producing E. coli from pigs and pig farm workers.

\begin{tabular}{|c|c|c|c|}
\hline Topoisomearase Gene & $\begin{array}{l}\text { Mutation Region and } \\
\text { Amino Acid Change }\end{array}$ & $\begin{array}{l}\text { Number of Pig Isolates }(n) \\
\qquad(\mathrm{N}=43)\end{array}$ & $\begin{array}{c}\text { Number of Human } \\
\text { Isolates }(n) \\
(\mathrm{N}=31)\end{array}$ \\
\hline \multirow{6}{*}{ gyrA } & Ser83 $\rightarrow$ Leu & 19 & 17 \\
\hline & Asp $87 \rightarrow$ Ans & 9 & 6 \\
\hline & Asp87 $\rightarrow$ Ala & & 1 \\
\hline & Asp87 $\rightarrow$ Gly & 1 & \\
\hline & Asp $87 \rightarrow$ His & & 1 \\
\hline & Asp87 $\rightarrow$ Tyr & & 2 \\
\hline \multirow{3}{*}{$\operatorname{parC}$} & Ser $80 \rightarrow$ Ile & 11 & 10 \\
\hline & Ser56 $\rightarrow$ Thr & 3 & \\
\hline & Ser57 $\rightarrow$ Thr & 1 & \\
\hline \multirow{3}{*}{ parE } & Ser $458 \rightarrow$ Ala & 1 & 4 \\
\hline & Iso335 $\rightarrow$ Thr & & 1 \\
\hline & Leu $416 \rightarrow$ Phe & & 1 \\
\hline
\end{tabular}

Abbreviation: Ser, Serine; Leu, Lecine; Asp, Aspartic acid; Ala, Alanin; Ans, Asparagine; Gly, Glycine; His, Histadine; Tyr, Tyrosine; Ile, Isoleucine; Thr, Threonine; and Phe, Phenilalaniene.

Three human isolates harboring the qnrS1 gene were phenotypically susceptible to NAL and CIP. Among pig isolates, 13 isolates harboring the qnrS1 gene also were phenotypically susceptible, while one isolate showing resistance to nalidixic acid had no resistance genotype. In summary, 18/43 ESBL-producing E. coli from pigs co-carried colistin and quinolone resistance genotypes (Figure 2), but four of these isolates did not show phenotypic resistance to quinolones. Four of the 31 human ESBL-producing isolates had resistance genotypes for colistin and quinolone, but one of the four (Ec_224) was phenotypically susceptible to quinolones.

\subsubsection{Resistance Genotypes to Other Antimicrobials}

Twenty aminoglycoside resistance genes were found in the 74 ESBL-producing E. coli isolates, of which 19 genes were present in pig isolates. The most common aminoglycoside resistance genes in the pig isolates were aadA1 (28), aph (3'-Ia (21), aph (6)-Id (21), aac (3)-IId (20), and aadA2 (17) (Table S3). Two pig isolates (Ec_168 and Ec_447) carrying one or two aminoglycoside resistance genes (aadA1 and/or aadA2) were susceptible to GEN and STR. The isolates from workers carried 11 aminoglycoside resistance genes, mainly aph (6)Id (12), aph (3'-Ib (11), and aadA1 (10) (Table S3). Four of them carried only one or two of the genes (aadA2 and/or aadA5) but were susceptible to GEN and STR, while one isolate (Ec_236) was resistant to STR but did not carry a resistance gene.

Analysis of the genetic background of resistance to sulfonamides revealed that sul1, sul2, and sul3 genes were present in both the human and pig isolates. The pig isolates mostly harbored sul3 (28), while sul1 was predominant in the isolates from workers (14). One human isolate (Ec_480) showed phenotypic sulphonamide resistance but did not contain any associated resistance gene. Trimethoprim resistance genes were found in 42 pig 
isolates and 23 human isolates. Genes detected included dfrA1, dfrA12, dfrA14, dfrA15, $d f r \mathrm{~A} 16, d f r \mathrm{~A} 17$, and $d f r \mathrm{~A} 27$. Six of these seven genes were found in the pig isolates with the dominant genes being $d f r A 12(n=20)$ and $d f r A 14(n=19)$. Five genes were present in isolates from workers, particularly dfrA17 $(n=9)$ and $d f r A 14(n=8)$. One pig isolate (Ec_388) and two human isolates (Ec_179 and Ec_368) showed phenotypic resistance to trimethoprim but no associated gene was detected (Table S3). Tetracycline resistance genes tet $(\mathrm{A})$, tet $(\mathrm{B})$, tet $(\mathrm{C})$, tet $(\mathrm{M})$, and tet $(\mathrm{X} 4)$ were found in 42 ESBL-producing E. coli from pigs and 22 human isolates (Table S3). tet (A) and tet (M) were the most dominant genes in both the pig and human isolates. One pig isolate (Ec_9) and one human isolate (Ec_219) were resistant to tetracycline but did not carry any tet genes, whereas the human isolate Ec_489 carrying tet (M) was phenotypically sensitive to tetracycline. Moreover, 37 of the pig isolates and 14 isolates from the workers carried chloramphenicol resistance genes, mainly floR and $\mathrm{cmlA} 1$ (Table S3), with floR present in 33 pig isolates and 10 human isolates, while $\mathrm{cmlA} 1$ was found in 24 pig isolates and five human isolates. Macrolide resistance genes detected were erm (B), $\ln u(\mathrm{~F}), \ln u(\mathrm{G}), \operatorname{mdf}(\mathrm{A}), \operatorname{mef}(\mathrm{B})$, and $m p h(\mathrm{~A})$, of which the $m d f$ (A) gene was present in all the 74 ESBL-producing E. coli isolates. Fosfomycin and rifampicin resistance genes were only found in a few pig isolates, with three isolates carrying the fosfomycin resistance gene fos $\mathrm{A} 3$. The rifampicin resistance genes aar 2 and aar3 were present in seven and two pig isolates, respectively (Table S3).

Metal resistance genes encoding resistance to copper, zinc, cobalt, and cadmium were found in all 74 ESBL-producing E. coli isolates (Table S5). Two pig isolates and four human isolates carried the metal resistance genes on plasmid contigs. Five pig isolates and ten human isolates carried the chromate transport protein ChrA on plasmids, encoding chromium resistance. Mercury resistance genes were found in 42 out of 43 pig isolates and 27 out of 31 human isolates, of which six pig isolates and two human isolates carried mercury resistance operon $(\mathrm{mer} C / \mathrm{merE} / \mathrm{merT})$ on plasmids.

\subsection{Plasmid Replicons in ESBL-Producing E. coli}

The isolates had a wide variation and number of plasmid replicons, with 23 plasmid replicons found in 39 pig isolates, ranging from one to six replicons per isolate (Table 3). The most dominant plasmid replicon in pig isolates was IncX1 types $(n=8)$. IncFIA (HI1), IncFIB (K), and IncY co-occurred in four isolates and the IncFII, IncFIB (AP001918), IncFIC (FII), IncN, and p0111 replicons occurred together in three isolates. Only three isolates out of the 43 isolates from pigs ( $7 \%$ ) were found to encode plasmid replicons from the same contigs as the ESBL genes. Of these, two isolates harbored $b l a_{\mathrm{CTX}-\mathrm{M}-55}$ on the same contig as IncX1 and one isolate harbored $b l a_{\mathrm{CTX}} \mathrm{M}-55$ on the same contig as the IncX2 replicon.

In the 31 ESBL-producing E. coli from human feces, 29 harbored plasmid replicons, varying from one to seven replicons per strain. The most frequent replicons were IncFIB (AP001918) $(n=12), \operatorname{IncFII}(n=6), \operatorname{IncFIA~}(n=5), \operatorname{IncFIA}(\mathrm{HI} 1)(n=5), \operatorname{IncFIB}(\mathrm{K})(n=5)$, IncFII (pCoo) $(n=4)$, and IncY $(n=4)$. Three of these isolates harbored ESBL genes

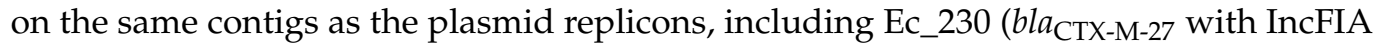
(HI1)), Ec_ 84 (bla $a_{\mathrm{CTX}-\mathrm{M}-24}$ with IncP1), and Ec_271 (bla as shown in Table 2 . Our data indicate that $b l a_{\mathrm{CTX}-\mathrm{M}-55}$ is likely associated with the IncX $(1 / 2)$ plasmid replicon types seen in pigs and humans, although this needs to be confirmed in additional isolates.

Table 3. Genomic characterization of ESBL-producing E. coli isolated from pig farm workers and pigs.

\begin{tabular}{|c|c|c|c|c|c|c|c|c|}
\hline Strain ID & MLST & Genome Size & GC\% & N50 & Phylogroup & Serotype & Virulence Genes & Plasmid Replicons \\
\hline Ec_1 1 * & ST5855 & $4,548,521$ & 51.12 & 21,866 & $\mathrm{~A}$ & $\mathrm{H} 38$ & ompT, terC & IncY \\
\hline Ec_3 ${ }^{p}$ & ST4956 & $4,647,525$ & 51.1 & 23,906 & B1 & H37 & $\operatorname{lpf} A, \operatorname{ter} C$ & ND \\
\hline Ec_6 ${ }^{p}$ & ST5870 & $4,899,146$ & 51.03 & 50,792 & A & O147:H40 & ast $A, \operatorname{ter} C, \operatorname{tra} T$ & $\begin{array}{c}\text { IncFIA (HI1), IncFIB (K), IncFII, } \\
\text { IncN, IncX1 }\end{array}$ \\
\hline Ec_7 $\mathrm{p}$ & ST877 & $4,728,224$ & 50.94 & 29,266 & B1 & O45:H10 & iss, $\operatorname{lpf} A$, ompT, terC, traT, & IncFII, IncR \\
\hline
\end{tabular}


Table 3. Cont.

\begin{tabular}{|c|c|c|c|c|c|c|c|c|}
\hline Strain ID & MLST & Genome Size & $\mathrm{GC} \%$ & N50 & Phylogroup & Serotype & Virulence Genes & Plasmid Replicons \\
\hline Ec_9p & ST10 & $4,873,762$ & 50.71 & 71,377 & A & $\mathrm{H} 32$ & terC & $\begin{array}{l}\text { IncFIA (HI1), IncFIB (K), } \\
\text { IncHI1A, IncHI1B (R27) }\end{array}$ \\
\hline Ec_21 ${ }^{p}$ & ST10 & $4,770,784$ & 50.89 & 72,227 & A & $\mathrm{O} 45: \mathrm{H} 45$ & kpsE, kpsMII, terC & IncFIA (HI1), IncFIB (K), IncX1 \\
\hline Ec_219 $\mathrm{h}^{*}$ & ST1163 & $4,905,795$ & 51.02 & 73,212 & G & $\mathrm{H} 23$ & $\begin{array}{c}\text { iss, } \operatorname{lpf} A, \operatorname{sen} B, \operatorname{chu} A, n e u C, \\
\operatorname{omp} T, \operatorname{ter} C, \operatorname{tra} T\end{array}$ & $\begin{array}{l}\text { Col156, IncFIA, IncFIB } \\
\text { (AP001918), IncFII }\end{array}$ \\
\hline Ec_309 P & ST10 & $4,820,803$ & 50.9 & 43,099 & $\mathrm{~A}$ & $\mathrm{H} 32$ & iss, $\operatorname{terC}$ & ND \\
\hline Ec_335 h & ST394 & $5,103,214$ & 50.52 & 72,314 & $\mathrm{D}$ & O17/O77:H18 & $\begin{array}{l}\text { aap, air, ast } A, \text { eilA, } \operatorname{lpf} A, \\
\text { chuA, } k p s E, \text { kpsMII_K1, } \\
\text { neuC, terC, traT }\end{array}$ & IncFII, IncFII (pHN7A8) \\
\hline Ec_314 P & ST2170 & $5,061,254$ & 50.7 & 43,068 & A & H51 & $\begin{array}{c}\text { cma, iss, } \operatorname{lpf} A, \text { fyuA, } h l y F, \\
\text { irp2, iucC, iut } A, \text { ompT, sit } A, \\
\text { terC, traT }\end{array}$ & $\begin{array}{l}\text { IncFIB (AP001918), IncFIB } \\
\text { (Plf82-PhagePlasmid), IncFIC } \\
\text { (FII), IncI1-I (gamma) }\end{array}$ \\
\hline Ec_340 h & ST345 & $4,880,303$ & 50.61 & 82,846 & B1 & O8:H21 (53) & $\operatorname{lpf} A, \operatorname{ter} C$ & IncI1, IncY \\
\hline Ec_116 P & ST3588 & $4,866,794$ & 50.99 & 23,092 & ND & O8:H7 & $\begin{array}{l}\text { iroN, iss, } \operatorname{lpf} A, \text { mchF, tsh, } \\
\text { cvaC, etsC, hlyF, iucC, iutA, } \\
\text { ompT, sit } A, \text { terC, traT }\end{array}$ & $\begin{array}{l}\text { IncFIB (AP001918), IncFIC } \\
\text { (FII), IncFII (pHN7A8), IncN }\end{array}$ \\
\hline Ec_224 h & ST196 & $5,127,497$ & 50.46 & 85,977 & B1 & O8:H7 & $\begin{array}{l}\text { iroN, iss, } \operatorname{lpfA}, \text { hlyF, ompT, } \\
\text { sit } A, \text { terC, traT }\end{array}$ & $\begin{array}{c}\text { IncFIA (HI1), IncFIB } \\
\text { (AP001918), IncFII (pCoo), } \\
\text { IncHI1A, IncHI1B (R27), IncI2, } \\
\text { p0111 }\end{array}$ \\
\hline Ec_123 P & ST218 & $4,902,464$ & 51 & 38,282 & A & $\mathrm{H} 23$ & $\operatorname{TerC}, \operatorname{TraT}$ & $\begin{array}{l}\text { IncFIA (HI1), IncFIB (K), IncFII, } \\
\text { IncN, IncX1 }\end{array}$ \\
\hline Ec_129 P & ST195 & $4,641,276$ & 50.92 & 27,405 & ND & $\mathrm{H} 4$ & $\operatorname{lpf} A, \operatorname{ter} C$ & ND \\
\hline Ec_228 h & ST68 & $4,995,853$ & 50.73 & 68,496 & $\mathrm{D}$ & $\mathrm{H} 6$ & $\begin{array}{l}\text { air, eilA, iss, } \operatorname{lpf} A, \operatorname{chu} A, \\
\text { kpsE, kpsMII, sit } A, \operatorname{ter} C\end{array}$ & $\operatorname{IncFIB~(K)~}$ \\
\hline Ec_130 P & ST48 & $4,963,599$ & 50.75 & 51,687 & B1 & O15:H11 & ast $A$, omp $T$, ter $C$ & IncN, IncX1, p0111 \\
\hline Ec_131 p & ST515 & $5,015,450$ & 50.61 & 73,216 & B1 & $\mathrm{H} 12$ & fyuA, irp2, terC & $\begin{array}{l}\text { IncFIA (HI1), IncHI1A, } \\
\text { IncHI1B (R27) }\end{array}$ \\
\hline Ec_274 P & ST278 & $4,936,780$ & 50.61 & 61,512 & B1 & $\mathrm{H} 7$ & fim $41 A, \operatorname{lpf} A, \operatorname{ter} C$ & IncFIB (pB171), IncFII, IncX1 \\
\hline Ec_230 h & ST351 & $5,181,680$ & 50.5 & 68,811 & B1 & O18:H7 & $\begin{array}{c}\text { cma, iroN, iss, cvaC, ets } C, \\
\text { hlyF, ompT, papC, sit } A, \operatorname{ter} C\end{array}$ & $\begin{array}{c}\text { IncFIA (HI1), IncFIB } \\
\text { (AP001918), IncFII, IncHI1A, } \\
\text { IncHI1B (R27) }\end{array}$ \\
\hline Ec_400 h & ST156 & $5,241,723$ & 50.5 & 42,932 & A & O76:H45 & $\begin{array}{c}\text { cma, ast } A, \text { hlyF, hra, iucC, } \\
\text { iut } A, \text { omp } T \text {, papC, sit } A, \\
\text { terC, traT }\end{array}$ & IncFIB (AP001918), IncFIC (FII) \\
\hline Ec_361 p & ST10 & $5,207,190$ & 50.7 & 75,974 & A & $\mathrm{O} 29: \mathrm{H} 10$ & ast $A, \operatorname{sit} A, \operatorname{ter} C$ & IncHI2, IncHI2A, IncQ1, IncY \\
\hline Ec_40 $45^{\mathrm{h}}$ & ST1286 & $5,984,169$ & 50.34 & 26,744 & B1 & O71:H32 & $\begin{array}{l}\text { aap, ast } A, \text { iha, } m c h B, m c h C, \\
m c h F, \text { fyu } A, \text { irp } 2, \operatorname{ter} C, \operatorname{traT}\end{array}$ & $\begin{array}{c}\text { IncB/O/K/Z, IncFII, IncFII } \\
\text { (pHN7A8) }\end{array}$ \\
\hline Ec_381 p & ST29 & $5,102,316$ & 50.59 & 73,837 & B1 & O123/186:H11 & $\begin{array}{c}\text { ast } A, \text { cif, eae, efa1, esp A, } \\
\text { esp } B, \text { espJ, iha, iss, katP, } \\
\text { lpf } A, \text { nle } A, \text { nleB, nleC, sep } A, \\
\text { tir, cea, iucC, iut } \text {, ompT, } \\
\text { terC, traT, fyuA, irp2 }\end{array}$ & $\begin{array}{l}\text { IncFIB (AP001918), IncFII } \\
\text { (pHN7A8), IncHI2, IncHI2A }\end{array}$ \\
\hline Ec_410 h & ST2170 & $5,023,174$ & 50.9 & 32,449 & B1 & H51 & $\begin{array}{c}\text { cma, iss, } \operatorname{lpf} A, \text { fyuA, } h l y F, \\
\text { irp2, iucC, iut } A, \text { ompT, sitA, } \\
\text { terC, traT }\end{array}$ & $\begin{array}{l}\text { IncFIB (AP001918), IncFIB } \\
\text { (pLF82), IncFIC (FII), IncI1 }\end{array}$ \\
\hline Ec_388 p & ST5229 & $4,859,334$ & 50.86 & 30,295 & ND & O177:H34 & $\begin{array}{l}\text { ast } A, \operatorname{lpf} A, \text { fyu } A, \operatorname{irp} 2, \text { iucC, } \\
\text { iut } A, \text { pap } C \text {, sit } A, \text { ter } A, \text { traT }\end{array}$ & $\begin{array}{l}\text { IncHI2, IncFIB (AP001918), } \\
\text { IncFII (pHN7A8), IncN }\end{array}$ \\
\hline Ec_155 P & ST165 & $4,879,514$ & 50.98 & 37,635 & ND & $\mathrm{H} 26$ & $\operatorname{terC}$ & IncQ1, IncR, IncX1, IncY \\
\hline Ec_170 h & ST1147 & $5,104,121$ & 50.7 & 91,960 & B1 & H35 & $\begin{array}{l}\text { aap, aat } A, \text { iha, iss, } \operatorname{lpf} A, \\
\text { fyu } A, \text { irp } 2, \text { ter } C, \operatorname{tra} T\end{array}$ & IncFIB (AP001918), IncFII (29) \\
\hline Ec_278 $\mathrm{P}$ & ST744 & $4,925,781$ & 50.7 & 103,452 & A & O162/89:H9 & $\begin{array}{l}\text { cma, cvaC, hlyF, iucC, iutA, } \\
\text { ompT, sitA, terC, traT }\end{array}$ & $\begin{array}{l}\text { IncFIB (AP001918), IncFIC } \\
\text { (FII), IncN, IncQ1, IncX4 }\end{array}$ \\
\hline Ec_172 h & ST69 & $5,391,031$ & 50.77 & 72,381 & $\mathrm{D}$ & O15:H18 & $\begin{array}{c}\text { air, eilA, iha, iss, lpfA, sat, } \\
\text { senB, chuA, fyuA, irp2, } \\
\text { iucC, iutA, kpsE, } \\
\text { kpsMII_K52, ompT, } \\
\text { papA_fsiA_F16, papC, sitA, } \\
\text { terC, traT }\end{array}$ & $\begin{array}{l}\text { Col156, IncB/O/K/Z, IncFIB } \\
\text { (AP001918) }\end{array}$ \\
\hline Ec_162 p & ST2170 & $4,901,850$ & 50.7 & 65,441 & B1 & O78:H51 & $\begin{array}{c}\text { cma, iss, } \operatorname{lpf} A, \text { fyuA, } h l y F, \\
\text { irp2, iucC, iut } A, \text { ompT, sit } A, \\
\text { terC, traT }\end{array}$ & $\begin{array}{l}\text { IncFIB (AP001918), IncFIC } \\
\text { (FII), IncI2 }\end{array}$ \\
\hline Ec_166 P & ST2954 & $4,803,218$ & 50.87 & 19,954 & ND & O160:H9 & air, chuA, terC & IncFIB (pLF82), p0111 \\
\hline
\end{tabular}


Table 3. Cont.

\begin{tabular}{|c|c|c|c|c|c|c|c|c|}
\hline Strain ID & MLST & Genome Size & GC\% & N50 & Phylogroup & Serotype & Virulence Genes & Plasmid Replicons \\
\hline Ec_232 h & ST167 & $4,956,803$ & 50.84 & 70,019 & A & $\begin{array}{c}\text { O-89 } \\
(162): H 10\end{array}$ & fyuA, irp $2, \operatorname{ter} C, \operatorname{traT}$, sit $A$ & $\begin{array}{l}\text { Col (BS512), IncFIA, IncFIB } \\
\text { (AP001918), IncFII }\end{array}$ \\
\hline Ec_168 P & ST2705 & $4,726,759$ & 50.8 & 35,199 & $\mathrm{~A}$ & $\mathrm{H} 10$ & omp T, TerC & p0111 \\
\hline Ec_179 h & ST5044 & $4,523,830$ & 50.79 & 93,960 & B1 & $\mathrm{H} 29$ & terC & ND \\
\hline Ec_283 P & ST1011 & $5,130,567$ & 50.55 & 61,007 & $\mathrm{D}$ & O8:H16 & $\begin{array}{c}\text { air, cma, eil } A, \text { iroB, iss, } \\
\text { chuA, cvaC, hlyF, iucC, } \\
\text { iut } A, \text { ompT, sit } A, \text { terC, traT }\end{array}$ & $\begin{array}{l}\text { IncFIB (AP001918), IncFIB } \\
\text { (pLF82), IncFIC (FII), IncX4 }\end{array}$ \\
\hline Ec_53 $\mathrm{p}$ & ST6775 & $4,825,481$ & 50.8 & 26,621 & B1 & O65:H49 & iss, $\operatorname{ter} \mathrm{C}$ & IncHI2, IncHI2A, IncX1 \\
\hline Ec_236 ${ }^{\mathrm{h}}$ & ST1294 & $4,511,999$ & 50.78 & 126,228 & A & O9:H30 & terC & $\mathrm{ND}$ \\
\hline Ec_57 p & ST165 & $5,115,907$ & 50.56 & 52,960 & A & $\mathrm{H} 27$ & terC & $\begin{array}{l}\text { IncHI2, IncHI2A, IncQ1, IncR, } \\
\text { IncX1 }\end{array}$ \\
\hline Ec_292 p & ST1121 & $4,893,986$ & 50.7 & 83,326 & A & $\mathrm{H} 48$ & Ast $A, \operatorname{lpf} A, \operatorname{ter} C$ & IncN, IncY \\
\hline Ec_416 ${ }^{\mathrm{P}}$ & ST10 & $4,680,174$ & 50.7 & 97,966 & A & $\mathrm{O} 25: \mathrm{H} 32$ & $\operatorname{ter} C$ & IncFIB (K) \\
\hline Ec_48 $48{ }^{\mathrm{h}}$ & ST34 & $4,650,258$ & 50.92 & 60,644 & A & H30 & $\operatorname{terC}$ & IncFIA (HI1), IncFIB (K) \\
\hline Ec_426 P & ST2935 & $4,875,048$ & 50.86 & 54,178 & A & $\mathrm{H} 32$ & ast $A, \operatorname{ter} C$ & IncP1, IncQ1, IncX1, IncY \\
\hline Ec_48 ${ }^{\mathrm{h}}$ & ST484 & $4,877,138$ & 50.57 & 68,561 & A & $\mathrm{H} 4$ & $\begin{array}{c}\text { aap, ast } A, \text { iss, } k p s E, k p s M I I \\
\text { ompT, terC, traT }\end{array}$ & IncFIC (FII), IncFII (pCoo) \\
\hline Ec_431 p & ST226 & $4,694,058$ & 51 & 45,154 & A & O15:H10 & fyuA, irp2, terC & ND \\
\hline Ec_489 h & ST34 & $5,015,072$ & 50.6 & 59,062 & A & $\begin{array}{c}\mathrm{O} 68 \\
(62): \mathrm{H} 30\end{array}$ & ast $A, \operatorname{Ter} C$ & $\begin{array}{c}\text { IncFIA (HI1), IncFIB (K), } \\
\text { IncHI1A, IncHI1B (R27), IncR, } \\
\text { IncX1 }\end{array}$ \\
\hline Ec_434 ${ }^{\mathrm{p}}$ & ST641 & $4,959,009$ & 50.7 & 29,769 & B1 & $\mathrm{H} 21$ & ast $A, \operatorname{lpf} A, \operatorname{ter} C$ & IncHI2, IncHI2A \\
\hline Ec_495 h & ST1722 & $5,038,475$ & 50.7 & 83,575 & $\mathrm{~F}$ & $\mathrm{H} 24$ & $\begin{array}{c}\text { air, eclb, eilA, iss, } \operatorname{lpf} A, \\
\text { chuA, fyuA, irp2, kpsE, sitA, } \\
\operatorname{terC}, \operatorname{traT}\end{array}$ & $\begin{array}{l}\text { Col (IMGS31), Col156, IncFIA, } \\
\text { IncFIB (AP001918), IncFII }\end{array}$ \\
\hline Ec_297 P & ST410 & $5,069,502$ & 50.6 & 48,156 & $\mathrm{C}$ & O33:H26 & iss, lpfA, ompT, TerC, traT & $\begin{array}{c}\text { IncFIA (HI1), IncFIB } \\
\text { (AP001918), IncFII (pHN7A8), } \\
\text { IncHI1A, IncHI1B (R27), IncI1 }\end{array}$ \\
\hline Ec_60 P & ST515 & $5,084,460$ & 50.9 & 65,609 & B1 & O128:H12 & fyuA, irp $2, \operatorname{ter} C, \operatorname{traT}$ & IncFIB (AP001918) \\
\hline Ec_8 $84^{\mathrm{h}}$ & ST48 & $4,947,358$ & 50.76 & 72,323 & A & $\begin{array}{c}\mathrm{O} 20 \\
(137): \mathrm{H} 45\end{array}$ & $\begin{array}{l}\text { cma, iroN, iss, katP, } m c h F, \\
\text { cvaC, hlyF, iucC, iut } A, \\
\text { ompT, sitA, terC, traT }\end{array}$ & IncFIB (AP001918), IncP1 \\
\hline Ec_63 $\mathrm{p}$ & ST515 & $4,812,948$ & 50.9 & 55,529 & B1 & O128:H12 & fyuA, irp2, terC & Col440II \\
\hline Ec_89 h & ST2040 & $4,932,400$ & 50.7 & 69,363 & A & $\mathrm{O} 159: \mathrm{H} 20$ & ast $A, \operatorname{lps} A, \operatorname{cib}, \operatorname{sit} A, \operatorname{ter} C$ & IncB/O/K/Z, IncFII (pCoo) \\
\hline Ec_67 p & ST6214 & $5,112,157$ & 50.9 & 56,813 & B1 & $\mathrm{O} 162 / 89: \mathrm{H} 10$ & $\begin{array}{l}\text { iss, nfaE, afaA, afaB, afaC, } \\
\text { afaD, afaE, fyuA, irp } 2, \text { iucC, } \\
\text { iutA, sitA, TerC, TraT }\end{array}$ & $\begin{array}{l}\text { IncFIB (AP001918), IncFII } \\
\text { (pRSB107) }\end{array}$ \\
\hline Ec_93 h & ST162 & $5,050,320$ & 50.6 & 72,932 & B1 & O8:H21 & $\begin{array}{c}\text { ast } A, \text { iss, } \operatorname{lpf} A, \text { hlyF, hra, } \\
\text { iucC, iutA, ompT, } \\
\text { papA-NEW, papC, sitA, } \\
\text { terC, traT }\end{array}$ & IncFIB (AP001918), IncFIC (FII) \\
\hline Ec_25 ${ }^{\mathrm{h}}$ & ST1722 & $4,823,623$ & 50.62 & 78,611 & $\mathrm{~F}$ & O1:H25 & $\begin{array}{c}\text { air, ast } A, \text { eilA, iss, } \operatorname{lpf} A, \\
\text { chuA, hra, kpsE, terC, yfc } V\end{array}$ & IncQ1 \\
\hline Ec_98 $\mathrm{h}$ & ST7153 & $4,746,746$ & 50.75 & 49,123 & A & O148:H30 & ast $A$, terC, traT & $\begin{array}{c}\text { IncFIA (HI1), IncFIB (K), IncX1, } \\
\text { IncY }\end{array}$ \\
\hline Ec_80 $\mathrm{p}$ & ST515 & $4,962,369$ & 50.9 & 38,744 & B1 & O128:H12 & fyuA, irp2, terC, traT & IncFIB (AP001918), p0111 \\
\hline Ec_271 h & ST1196 & $4,931,876$ & 50.84 & 129,873 & B1 & $\mathrm{O} 29: \mathrm{H} 8$ & $\begin{array}{c}\text { cma, } \operatorname{lpfA} \text {, cea, cvaC, } h l y F, \\
\text { iucC, iut } A, \text { ompT, sit } A, \operatorname{ter} C, \\
\text { traT }\end{array}$ & IncFIB (AP001918), IncFIC (FII) \\
\hline Ec_81 $\mathrm{P}$ & ST877 & $4,744,811$ & 50.8 & 59,541 & B1 & $\mathrm{O} 28 \mathrm{ac} / \mathrm{O} 42: \mathrm{H} 32$ & $\operatorname{lpf} A$, ompT, $\operatorname{Ter} C$ & $\begin{array}{l}\text { IncFIB (AP001918), IncFIA } \\
\text { (HI1), IncFIB (K), Inc1 }\end{array}$ \\
\hline Ec_447 P & ST4956 & $4,805,635$ & 50.9 & 54,620 & B1 & O156:H37 & $\operatorname{lpf} A, \operatorname{ter} C, \operatorname{tra} T$ & $\begin{array}{l}\text { IncHI2, IncFIB (AP001918), } \\
\text { IncFIC (FII) }\end{array}$ \\
\hline Ec_498 h & ST10 & $4,743,370$ & 50.91 & 77,783 & A & $\begin{array}{c}\text { O9 } \\
(89,162): \mathrm{H} 9\end{array}$ & sit $A$, ter $C$, traT & $\begin{array}{l}\text { IncFIA, IncFIB (AP001918), } \\
\text { IncFII }\end{array}$ \\
\hline Ec_83 P & ST6018 & $4,752,755$ & 50.79 & 69,914 & A & O8:H11 & ompT, ter C & IncX1, p0111 \\
\hline Ec_442 P & ST101 & $5,046,847$ & 50.26 & 71,098 & B1 & O118:H21 & $\begin{array}{c}\text { cma, iss, } \operatorname{lpf} A, \text { cvaC, } h l y F, \\
\text { iucC, iut } A, \text { ompT, sit } A, \operatorname{ter} C, \\
\text { traT }\end{array}$ & $\begin{array}{l}\text { IncFIB (AP001918), IncFIC } \\
\text { (FII), IncFII (29), IncI2 }\end{array}$ \\
\hline Ec_452 P & ST515 & $4,878,968$ & 51 & 42,049 & B1 & O128ac:H12 & fyuA, irp $2, \operatorname{terC}, \operatorname{traT}$ & IncFIB (AP001918), IncX4 \\
\hline
\end{tabular}


Table 3. Cont.

\begin{tabular}{|c|c|c|c|c|c|c|c|c|}
\hline Strain ID & MLST & Genome Size & GC\% & N50 & Phylogroup & Serotype & Virulence Genes & Plasmid Replicons \\
\hline Ec_500 h & ST2040 & $4,895,059$ & 50.77 & 26,675 & A & O159:H20 & ast $A, \operatorname{lpf} A, \operatorname{cib}$, sit $A, \operatorname{ter} C$ & IncB/O/K/Z, IncFII (pCoo) \\
\hline Ec_457 P & ST1121 & $4,935,661$ & 50.57 & 131,601 & A & $\mathrm{H} 48$ & $\operatorname{lpf} A, \operatorname{ter} C$ & IncX4, IncY \\
\hline Ec_503 ${ }^{\mathrm{h}}$ & ST192 & $4,868,610$ & 50.73 & 79,109 & B1 & $\begin{array}{c}\mathrm{O} 124 \\
(164): \mathrm{H} 34\end{array}$ & iss, $\operatorname{lpf} A$, omp $T$, terC & IncX2, p0111 \\
\hline Ec_511 h & ST38 & $5,198,810$ & 50.66 & 73,146 & $\mathrm{D}$ & O1:H15 & $\begin{array}{c}\text { air, iha, iss, sat, sen } B, \text { chuA, } \\
\text { fyuA, hra, irp2, iucC, iut } A, \\
\text { kpsEkpsMII_K5, papA_F43, } \\
\text { terC, traT, kpsE }\end{array}$ & $\begin{array}{c}\text { Col156, IncFIA, IncFIB } \\
\text { (AP001918), IncFII (pRSB107) }\end{array}$ \\
\hline Ec_467 P & ST4238 & $4,663,515$ & 50.71 & 92,493 & A & $\mathrm{H} 2$ & capU, terC & IncFIB (K) \\
\hline Ec_514 ${ }^{\mathrm{h}}$ & ST38 & $5,084,740$ & 50.7 & 54,474 & $\mathrm{D}$ & O86:H18 & $\begin{array}{l}\text { air, ast A, eilA, iss, afaD, } \\
\text { chuA, fyuA, irp2, kpsE, } \\
\text { kpsMII_K5, terC }\end{array}$ & IncL/M (pOXA-48) \\
\hline Ec_472 P & ST877 & $4,948,850$ & 50.7 & 72,864 & B1 & $\mathrm{H} 10$ & $\operatorname{lpf} A, \operatorname{omp} T, \operatorname{TerC}, \operatorname{traT}$ & IncFII (pCoo), IncI1, IncX1 \\
\hline Ec_517 h & ST1141 & $5,068,761$ & 50.82 & 55,942 & B1 & O13:H11 & ast $A, \operatorname{ter} C$, traT & $\begin{array}{l}\text { Col (BS512), Col (IRGK), } \\
\text { IncFIB (K), IncR, IncY }\end{array}$ \\
\hline Ec_519 h & ST6253 & $5,382,277$ & 50.8 & 35,706 & $\mathrm{D}$ & H15 & $\begin{array}{c}\text { aap, air, ast } A, \text { capU, eat } A, \\
\text { eilA, chuA, kpsE, } \\
\text { kpsMII_K5, terC, traT }\end{array}$ & IncFII \\
\hline
\end{tabular}

* P: Pig isolates; h: Human isolates.

\subsection{Genetic Diversity of ESBL-Producing E. coli}

The MLST analysis revealed 53 different sequence types among the 74 ESBL-producing E. coli. The sequence types were also different between the pig and human isolates from the same farms. The main STs in the pig isolates were ST10 and ST515, with five isolates in each (Table 3). ST10 isolates belonged to phylogroup A and carried the ESBL genes $b l a_{\mathrm{CTX}-\mathrm{M}-55}$ (3), bla ${ }_{\mathrm{CTX}-\mathrm{M}-27}(1)$, and $b l a_{\mathrm{CTX}-\mathrm{M} 15}$ (1). The isolates of ST515 belonged to phylogroup B1 and

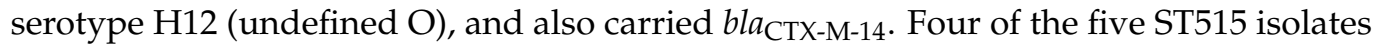
had the same O group (O128). Three ST877 isolates (phylogroup B1) carried bla $a_{\mathrm{CTX}-\mathrm{M}-55}$, bla $_{\mathrm{CTX}-\mathrm{M}-24}$, or bla $a_{\mathrm{CTX}-\mathrm{M}-15}$ but originated from three different districts in the Bac Ninh Province. Two isolates belonged to ST1121 and both carried bla from workers revealed 27 different sequence types. Sequence types ST1722, ST2040, ST34, and ST38 were found in two isolates each.

Only three sequence types (ST10, ST48, and ST2170) were shared between the pig and human isolates, but the isolates displayed different resistotypes (Figure 2).

In general, our data revealed that there was high heterogeneity among the ESBLproducing E. coli from the pigs and workers with respect to phylogroups, serotypes, and sequence types. In a simmer manner, the phylogenetic analysis also revealed a wide variation with up to 4046 SNPs between the 74 strains (Figure 2, Table S4). Six heterogeneous clades of different sequence types and serotypes appeared with more than 1000 SNPs between the isolates. There was no significant difference of distribution between the pig and human isolates in each cluster $(p>0.05)$. Although each of the clusters contained mixed strains, i.e., human and pig isolates, the strains did not often belong to the same STs, as described above, and they also showed differential status in the resistance genes; for instance, pig isolates harbored $m c r$ genes, which were lacking in the human isolates (Figure 2).

Some STs appeared to be genetically narrow, associated to only one of the two hosts in the current study. For example, strains of ST515 from different pig samples only differed by 1 to 3 SNPs and this ST was only found in samples from pigs. In a similar manner, the isolates which belonged to ST1722 only had 10 SNPs between them and were only found in human samples. The human isolates of ST2040 (last cluster in Figure 2) were identical and pig isolates of both ST1121 and ST4956 strains were also closely related with 5-6 SNPs.

The human isolates Ec_410 and Ec_517 were closely related to the pig isolate Ec_162, which only differed by 24 SNPs (Figure 2, Table S4). These three strains, however, were isolated from three different farms with no apparent epidemiological association. 


\subsection{Virulence Genes and Serotypes}

The most frequent virulence determinants associated with extra-intestinal pathogenic E. coli (ExPEC) were as follows, in descending order: $\operatorname{traT}(36), \operatorname{ompT}(26), \operatorname{sit} A$ (24), fyuA (19), irp2 (19), iutA (15), iucC (15), hlyF (13), chuA (12), kpsMII/E (10), cvaC (7), papA/C (6), $h r a(4), a f a A / B / C / D / E(2), y f c V(2), c i b(2), e t s C$ (2), and neuC (2). All these genes were evenly distributed between the human and porcine isolates except for the UPEC $\mathrm{HM}_{\text {-related }}$ chuA, which was found in ten human isolates and two pig isolates, and the ExPEC $\mathrm{JJ}_{\mathrm{JJ}}$-associated $\mathrm{kpsMII/E}$, which was found in eight human isolates and two pig isolates. hra was only found in four human isolates (Table 3). Three strains (Ec_172 ${ }^{\mathrm{h}}$, Ec_511 $\mathrm{h}$, and Ec_514 ${ }^{\mathrm{h}}$ ) could be classified as uropathogenic E. coli $\left(\mathrm{UPEC}_{\mathrm{HM}}\right)$ and ExPEC $\mathrm{CJJ}_{\mathrm{JJ}}$. Two human isolates (Ec_495 and Ec_255) could be classified as $\mathrm{UPEC}_{\mathrm{HM}}$. These five isolates were of different serotypes (Table 3). Virulence determinants not associated with ExPEC were found in more than two isolates and were as follows, in descending order: $\operatorname{lpf} A$ (31), iss (26), ast $A$ (20), air (10), cma (10), eilA (8), iha (5), aap (5), and senB (3). These genes were evenly distributed between isolates of porcine and human origin, except lpfA (20 of 31), iss (19 of 26), ast A (12 of 20), and cma (7 of 10), which were found more often in the porcine rather than human isolates. aap was only found in five human isolates (Table 3). Only one isolate (Ec381 P) of serotype O123/186:H11 harbored the eae gene allele (encoding intimin), as well as harbored 13 genes (ast $A, \operatorname{cif}, \operatorname{efa} 1, \operatorname{esp} A, \operatorname{esp} B, \operatorname{espJ}$, iha, katP, lpfA, nleA, nleB, nleC, and tir) usually associated with the attaching and effacing of both E. coli (AEEC) and ExPEC-related genes iucC, iutA, ompT, traT, fyuA, and irp2.

\section{Discussion}

ESBL-producing E. coli from pigs in Vietnam was found to differ genetically from ESBL E. coli obtained from farm workers. A high number of SNPs were detected between the isolates and together with a wide diversity in sequence types, serotypes, phylogroups, resistance genes, and plasmid replicon types, the results suggest a highly heterogeneous population with little evidence of transmission of ESBL-producing E. coli between the pigs and workers. Only one ESBL-producing E. coli was isolated from each fecal sample and analyzed by WGS, and we cannot rule out that pigs and farmers share strains, which are present in very low concentrations. The virulence gene profiles also suggested that the ESBL-producing E. coli analyzed were primarily commensal bacteria, which corroborates findings in a related investigation of pig farms in the same study area [10]. The present study did not compare E. coli from pork (food) to E. coli from humans and we cannot rule out that some of the human isolates originated from pigs. However, our study seems to rule out that direct contact with pigs at the farm level is a main route of exposure to ESBL E. coli. A similar situation was previously documented when E. coli from different livestock were shown to be of distinct lineages compared to the isolates collected in humans [17].

Five strains from workers could be classified as either $\mathrm{UPEC}_{\mathrm{HM}} / \mathrm{ExPEC}_{\mathrm{JJ}}$ or $\mathrm{UPEC}_{\mathrm{HM}}$. This classification has been derived by comparing limited sets of virulence genes with epidemiological and infection model data, and could indicate extra-intestinal virulence potential in the form of urinary tract infection (UTI) and/or bacteremia $[18,19]$. We found that the ESBL genes were present in a wide variety of E. coli STs and that some STs appeared to be more restricted to isolates as specific hosts [20]. ST69 ExPEC $\mathrm{CJJ}_{\text {J }}$ has been isolated from cases of bacteremia in Spain and France [21]. Two of our isolates were ST38, which has been described as an evolving enteroaggregative E. coli (EAEC) in the United Kingdom [22], but we did not identify the $a g g R$ genes defining EAEC. These strains were only found in workers, which could indicate that they are host-specific. Similar to our study, commensal E. coli from pigs in Denmark carried ST10 as the most common sequence type [23]. The dominance of the same ST type in pig isolates from Vietnam strains and Europe could suggest that this ST type is widespread in E. coli from pigs, although further studies are needed to determine this.

A study of poultry farms in the Red River Delta of Northern Vietnam showed that $83.1 \%$ of farm workers and $74.1 \%$ of chickens carried ESBL-producing E. coli [24], whereas 
a study of small-scale poultry farms and farming households in Southern Vietnam found that $31.4 \%$ of farmers and $14.7 \%$ of chickens carried ESBL-producing E. coli [16]. ESBLproducing $E$. coli has also been found in $31 \%$ of pork meat and $73.5 \%$ of asymptomatic resident volunteer workers at a local wholesale market in Central Vietnam [25]. Other studies confirm that the general Vietnamese population seems to have frequent carriage of ESBL-producing E. coli among residents in Thai Binh Province in Northern Vietnam, showing a prevalence of $61.2 \%$ ESBL-producing E. coli [26], while 51\% (111/198) of residents in suburban Hanoi harbored ESBL-producing E. coli [27]. Our findings confirm that the prevalence of ESBL-producing E. coli in people and animals are high in Vietnam.

The ESBL genes found in most of the pig isolates belonged to bla $a_{\mathrm{CTX}-\mathrm{M}}$ groups 1 (CTX-M15, CTX-M27, and CTX-M55) and bla CTX-M group 9 (CTX-M14 and CTX-M65). This confirms findings from previous studies that bla $a_{\mathrm{CTX}-\mathrm{M}}$ group 1 and 9 are predominant ESBL genes in pig farms and in both pork and retail markets [10], as well as in beef, chicken, and fish $[13,28,29]$ in Vietnam. CTX-M55 was the most common gene detected in ESBLproducing E. coli from pork sold at wholesale markets in Central Vietnam [25] and this was the most common gene in the pig isolates in our study. All our ESBL-producing

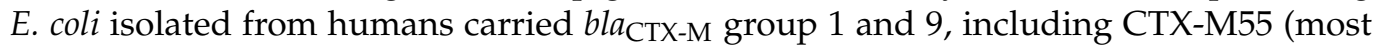
common), CTX-M27, CTX-M15, CTX-M14, CTX-M24, and CTX-M65; notably, these are genes which were also carried by ESBL-producing E. coli isolated from the pork and workers at wholesale markets, from urinary tract infection patients in Central Vietnam [25], and from both poultry and poultry farmers (CTX-M55) [16]. Together these data show that bla $a_{\text {CTX-M }}$ genes are the dominant ESBL genotypes circulating in Vietnam and are widely distributed in humans, livestock, and foods in different parts of the country.

One of the emerging concerns regarding ESBL-producing E coli is the co-occurrence of colistin and quinolone resistance, and many of our strains, particularly those from pigs, showed such a resistotype. Colistin is a last-resort drug for the treatment of multidrugresistant bacteria such as Acinetobacter spp. and Pseudomonas aeruginosa, and the spread of ESBL-producing bacteria harboring $m c r$ genes are of serious concern [30]. Of particular concern, many isolates showed mobile colistin resistance ( $m c r-1$ and $m c r-3)$ and plasmidmediated quinolone resistance ( $q n r S 1$ and $q n r \mathrm{~B} 4)$ genotypes. Quinolone and colistinresistant ESBL-producing E. coli have been reported in Japan, e.g., where a CTX-M-27 and CTX-M-14-producing and ciprofloxacin-resistant E. coli of the $\mathrm{H} 30$ sub-clonal group within ST131 was implicated in a Japanese regional ESBL epidemic [31-33]. The high level of quinolone resistance in the ESBL-producing E. coli found in both pigs $(64.1 \%)$ and farm workers (30.2\%) is in accordance with the findings in Southern Vietnam, e.g., where $42.7 \%$ to $62.7 \%$ ESBL/AmpC E. coli were resistant to quinolones [28,29]. Our results suggest that one mutation and presence of a $q n r$ gene could be sufficient to produce phenotypic quinolone resistance, which is in agreement with previous studies of ESBL-producing E. coli $[31,34]$. Quinolones (enrofloxacin and norfloxacin) are frequently used for the prevention and treatment of diarrhea in piglets in Vietnam [35]. It has been reported that quinoloneresistant E. coli have spread before the acquisition of ESBL genes [36]. This could explain the high level of quinolone resistance in the ESBL-producing E. coli found in our study. Our sequence data did not allow for concluding that $\mathrm{mcr}$ genes, along with quinolone and ESBL genes, were located on the same contigs as the plasmid replicons most likely because of the short reads-sequencing platform used. Nevertheless, the contigs harboring each of these genes yielded only plasmid hits in a preliminary Blast search, which may indicate they are transferable (data not shown). Our findings corroborate that $m c r-1$ and $m c r-3$ genes are frequently isolated in E. coli from pigs and humans in Vietnam [26,29,37,38]. Similar observations have been reported for E. coli originating from chickens in Vietnam [3]. The frequent finding of colistin and quinolone-resistance in ESBL-producing E. coli in pigs may pose a food safety concern and the level of transfer from pigs should be determined in order to assess whether they are implicated in human disease.

A few discrepancies were observed in the genotypic and phenotypic resistance. For instance, the lack of colistin resistance in the human isolate Ec309 could be attributed 
to the absence of any mor genes in the genome of this isolate, which only harbors the $p m r \mathrm{~B}$ mutation. Similarly, the presence of $q n r \mathrm{~S} 1$ alone was not enough to yield resistance to nalixidic acid and ciprofloxacin in the strains lacking the required mutations on the quinolone resistance-determining region.

Commensal E. coli are less studied than pathogenic E. coli; they are usually genetically distinct [23] and often harbor various resistance genotypes with the potential to be horizontally transferred to bacterial pathogens through the mobile genetic elements they contain, therefore representing a public health risk [26]. Opportunistic infections caused by the antimicrobial-resistant commensals, themselves, may also occur. Our data show that multiple resistance genes, along with ESBL genes, $m c r$ genes, and quinolone resistance genes, are harbored across diverse E. coli lineages of predominantly commensals types and are epidemiologically unrelated. Similar observations of high frequency drug resistance in commensal E. coli have been reported in ESBL strains in Poland [39]. The public health relevance of our commensal E. coli is further emphasized by the fact that they were predominately MDR [40], which is in agreement with previous studies documenting that ESBL-producing E. coli, whether commensal or pathogenic, are MDR [3,29,41,42]. Fifty-five of the farms studied bought piglets from other farms or pig companies, and the ESBLproducing E. coli found in mainly finisher pigs may have originated from such sources. Even in farms with a low use of antimicrobials, MDR E. coli are frequent probably because the strains circulating in the production system have been subjected to high selective pressure for a long time. Thus, weaned piglets from Danish pig farms not using antimicrobials have been shown to contain a high diversity of MDR commensal E. coli [23]. Various studies have documented the frequent use of third generation cephalosporin, quinolones, and colistin in humans and livestock in Vietnam [7,9,35,43], and that a high number of such antimicrobial products are easily accessible over the counter often without the need for medical and veterinary prescriptions [44,45]. However, it remains to be determined to what extent the high levels of antimicrobial resistance at our pig farms were associated with actual antimicrobial use or other driving factors.

There is an association between metals resistance and the occurrence of antimicrobial resistance, where exposure to metals such as zinc oxide is often applied to control diarrhea in weaning pigs and copper added to feed for growth promotion can co-select for antimicrobial bacterial resistance [46-48]. The MDR ESBL-producing E. coli characterized in this study contained a number of metal resistance genes, which may be associated with the high level of antimicrobial resistance. Twenty out of 74 ESBL-producing E. coli isolates carried heavy metal resistance genes and ESBL genes located on plasmid(s). Resistance to metals and antimicrobials may be associated as these are frequently located on the same mobile elements [49]. The abundance of heavy metal resistance genes on plasmids found in this study may contribute to the dissemination of ESBL genes. Unfortunately, we do not have sufficient data on the antimicrobial and metal use from the pig farms to evaluate to what extent the antimicrobial resistance found was associated with antimicrobial and metal exposures.

\section{Materials and Methods}

\subsection{Collection of Fecal Samples}

Fecal samples were collected from pigs and workers at 116 pig farms in Bac Ninh Province in Northern Vietnam. The farms varied in size and were randomly selected from a list of pig farms provided by veterinary authorities in the province. A total of 116 composite pig manure samples and 94 fecal samples from healthy farm workers were collected. A sample was collected from the top of fecal piles on the floor with a spoon from three locations within each pig pen, after which samples were pooled. A composite fecal sample with a weight between $100 \mathrm{~g}$ and $200 \mathrm{~g}$ which included feces from the different pig pens was collected in each farm. A maximum of three pig pens per farm were sampled. Each pen was randomly chosen to represent the pig type (sow, piglet, and finisher) in a total of 61 farms that produced their own piglets and raised them until slaughter. An approximately $20 \mathrm{~g}$ 
fecal sample was self-collected by one worker during defecation using sterile gloves on the same day as the pigs were sampled. Fecal samples were placed in labelled sterile plastic bags and immediately transported to the laboratory at the National Institute of Veterinary Research in Hanoi, at which they were analyzed the same day. Both farm owner and farm workers gave consent orally and understood that he/she could withdraw from the study at any time and would be anonymous in the reporting of the results. The study protocol was approved by the Ethical Committee of the National Institute of Nutrition in Hanoi (certificate number 04/VDD-QLKH).

\subsection{Sampling and Isolation of E. coli}

Ten grams of fecal sample was mixed with $90 \mathrm{~mL}$ of Peptone Buffered Saline containing $0.1 \%$ peptone (Himedia, Mumbai, India) and $0.85 \% \mathrm{NaCl}$ in a sterilized plastic bag, and was homogenized in a stomacher. One $10-\mu \mathrm{L}$ loop of the dilution was spread onto MacConkey agar (Merck, Darmstadt, Germany) plates with and without $2 \mathrm{mg} / \mathrm{L}$ of cefotaxime (breakpoint concentration according to EUCAST [50]) and was incubated at $37^{\circ} \mathrm{C}$ for $24 \mathrm{~h}$ for the selection of E. coli colonies [51]. Up to five presumptive E. coli colonies (red, smooth, round, $>2.5 \mathrm{~mm}$ diameter) that showed bacterial growth were selected from each plate with and without cefotaxime (Sigma Aldrich, St Louis, MO, USA) [51]. All colonies were selected if less than five colonies appeared on the MacConkey agar with $2 \mathrm{mg}$ cefotaxime. Confirmatory biochemical testing for $E$. coli included glucose (+), lactose $(+)$, gas $(+), \mathrm{H}_{2} \mathrm{~S}$ $(-)$, indole $(+)$, urease $(-)$, Voges-Proskauer $(-)$, methyl red (+), and citrate $(+)$ [51]. E. coli ATCC 25,922 was used as reference strain for quality control. Confirmed isolates were purified on blood agar plates and stored at $-80^{\circ} \mathrm{C}$ in Eppendorf tubes containing Brain Heart Infusion broth (CM1135; Oxoid, Basingstoke, UK) with 10\% glycerol.

\subsection{Antimicrobial Susceptibility Testing}

One colony confirmed as E. coli was randomly selected on agar plates with and without cefotaxime per fecal sample and was tested for susceptibility to: ampicillin (AMP, $10 \mu \mathrm{g}$ ), gentamycin (GEN, $10 \mu \mathrm{g}$ ), trimethoprim (TMP, $5 \mu \mathrm{g}$ ), tetracycline (TET, $30 \mu \mathrm{g}$ ), streptomycin (STR, $10 \mu \mathrm{g}$ ), nalidixic acid (NAL, $30 \mu \mathrm{g}$ ), sulfonamides (SUL, $300 \mu \mathrm{g}$ ), ciprofloxacin (CIP, $5 \mu \mathrm{g}$ ), ceftriaxone (CRO, $30 \mu \mathrm{g}$ ), cefoxitin (FOX, $30 \mu \mathrm{g}$ ), ceftiofur (EFT, $30 \mu \mathrm{g}$ ), and amoxicillin-clavulanic (AMC, $30 \mu \mathrm{g}$ ) by the Kirby-Bauer disc diffusion test according to the CLSI standard procedure [52]. Antimicrobial discs were from the Oxoid company (Basingstoke, UK). Isolates which were resistant to $\mathrm{CRO}$ were also tested for ESBL production by the modified double-disk synergy test (amoxicillin/clavulanic acid, 20/10 $\mu \mathrm{g}$ ) [53]. The interpretation of inhibition zones was performed according to CLSI criteria and the isolates showing intermediate resistance were categorized as susceptible to avoid overestimation of resistance. Micro-broth dilution assay was done to confirm colistin (COL) resistance, using dilutions of colistin sulphate from $32 \mu \mathrm{g} / \mathrm{mL}$ to $0.25 \mu \mathrm{g} / \mathrm{mL}$ (Sigma Aldrich, St Louis, MO, USA). E. coli ATCC 25,922 was used as a control strain (MIC of colistin: 0.25-2 $\mu \mathrm{g} / \mathrm{mL}$ ). Multidrug resistance (MDR) was defined when an isolate was not susceptible to at least one antimicrobial in at least three of the tested antimicrobial classes [40]. Prob.test in R studio was used to compare differences between the AMR proportion of two populations.

\subsection{Whole-Genome Sequencing and Sequence Analysis}

Two criteria were used to select confirmed ESBL-producing E. coli isolates from MacConkey agar plates with cefotaxime for whole-genome sequencing (WGS). One human and one pig ESBL-producing E. coli from 26 farms that had ESBL-producing isolates in the fecal samples from both hosts were selected $(n=52)$. ESBL-producing E. coli isolated from pigs and humans in the remaining farms were selected for WGS if they had different resistance profiles compared to the those from the 26 farms described above. Thus, a total of 74 ESBL-producing E. coli isolates, including 43 pig isolates and 31 human isolates, were selected. There was less human E. coli compared to the pig isolates because some human fecal specimens did not yield any E. coli colonies on the MacConkey agar plates containing 
cefotaxime. DNA was extracted by using an automated Maxwell DNA extraction system following the manufacturer's instruction (Promega Maxwell RSC, Maldison, WI, USA). The concentration of the extracted DNA was determined using NanoDrop. The DNA quality was checked following electrophoresis in a $1 \%$ agarose gel. The genomic DNA was sequenced on an Illumina pair-end sequencing platform using the Illumina Nextera XT and MiSeq reagent kit v.3 protocol. The paired-end raw reads were assembled using Spades 3.9 [54] and were quality checked using Quast [55]. The sequence reads have been submitted to the European Nucleotide Archive with the accession number PRJEB37980. Assembled sequences were analyzed using tools from the servers of the Center for Genomic Epidemiology (CGE) (https:/ / cge.cbs.dtu.dk/services / (accessed on 15 June 2020) for multi-locus sequence types using MLST 2.0. Antimicrobial resistance genes using ResFinder 4. Virulence determinants were assessed in the genomes using VirulenceFinder 2.0 from CGE, with default settings coupled with the BLAST algorithms through MyDbFinder (https: / / cge.cbs.dtu.dk/services / (accessed on 15 June 2020) using new curated databases of Extra-intestinal pathogenic E. coli genes (ExPEC) and eae genes for the attaching and effacing of E. coli (AEEC) [56]. The presumptive classification of E. coli isolates used two definitions, which classify isolates as (1) ExPEC JJ $_{\mathrm{J}}$ if positive for two or more of papAH and/or papC (P fimbriae), sfa/focDE (S and F1C fimbriae), afa/draBC (Dr-binding adhesins), iut $A$ (aerobactin siderophore system), and kpsM II (group 2 capsules) [18], and as (2) uropathogenic E. coli (UPEC $\mathrm{HM}_{\mathrm{HM}}$ ) if positive for two or more of chuA (heme uptake), fyuA (yersiniabactin siderophore system), vat (vacuolating toxin), and $y f_{c} V$ (adhesin) [19].

Metal resistance genes encoding resistance to copper, cobalt, mercury, zinc, cadmium, magnesium, and chromium were analyzed through the subsystem annotation in RAST [57]. Resistance to tellurite was detected by using BLASTn with the tellurite resistance genes tehA_NC_000913.3, tehB_M74072.1, and telluECs2035_EU901290.1. The detergent-resistant phospholipase A and pldA (NC_003198.1) as well as the quaternary ammonium compound efflux qacEdelta (NG_048042.1) were also searched for in the genomes using Blast.

The presence of plasmid replicons was determined using PlasmidFinder and the serotypes were determined using SerotypeFinder 2.0. Strains, in which the $\mathrm{O}$ antigen was not detected in the initial analysis, were subjected to a BLAST alignment using the O serotype database for confirmation. Enterobase (http://enterobase.warwick.ac.uk/ (accessed on 20 June 2020) was used to determine the phylogroups of the strains and to confirm both the serotypes and MLST findings. A phylogenetic analysis was performed with the 74 isolates using the pipeline CSI phylogeny 1.4 tool from the servers of the Center for Genomic Epidemiology (CGE) [58] with automate default settings using E. coli K12 substrain.MG1655 (accession number U00096) as reference. This built a consensus core-genome tree and generated a Newick tree file, along with the multiple alignment of the core-genomes' SNPs. The obtained tree was annotated in Interactive Tree Of Life iTOL v3 [59] and was interpreted along with the SNP (single nucleotide polymorphism) values between the genomes. Prob.test in $\mathrm{R}$ studio was used to compare the difference distribution between the pig and human isolates in each phylogenic cluster.

\section{Conclusions}

The results from our genetic diversity and phylogenic analysis of ESBL-producing E. coli showed that ESBL-producing E. coli from pigs were different from the ESBLproducing E. coli found in pig farm workers on the same farms. The highly heterogeneous population of commensal MDR ESBL-producing E. coli carrying plasmid-associated colistin and quinolone resistance genes, as well as carrying detergent and metal resistance genes, represent a potential source and possible hotspot for the horizontal transmission of antimicrobial resistance. Our findings confirm that the prevalence of ESBL-producing E. coli in people and animals is high in Vietnam. A main conclusion is that the direct contact with pigs may not be a likely contributor to the carriage of ESBL E. coli in farm workers. This does not rule out that pig strains can be transmitted to humans via the food chain and that the role of food from animal origin in the transmission of ESBL-producing E. coli to humans 
should be assessed. Moreover, actual antimicrobial use and other pig farm management factors impacting the emergence and maintenance of the antimicrobial resistance in pigs remains to be determined.

Supplementary Materials: The following are available online at https:/ /www.mdpi.com/article/ 10.3390/antibiotics10101165/s1, Table S1: Resistance profiles of indicator E. coli isolated from pigs and farm workers; Table S2: Resistance profiles of ESBL-producing E. coli isolated from pigs and farm workers; Table S3: Phenotypic and genotypic characteristic of ESBL-producing E. coli isolated from pigs and farm workers; Table S4: Matrix of main SNPs of ESBL-producing E. coli isolated from pigs and farm workers; and Table S5: Metal and detergent resistance genes of ESBL-producing E. coli isolated from pigs and farm workers.

Author Contributions: Authors A.D. and J.E.O. conceptualized and supervised the study. D.T.Q.T. performed the sampling, preliminary isolation, and antimicrobial sensitivity testing. Y.M.G.H. and D.T.Q.T. performed the whole-genome sequencing and bioinformatics analysis, and produced the first draft of the manuscript. Y.M.G.H. and F.S. interpreted the genomic data. G.T.H.T. and N.T.T. participated in the sampling and preliminary isolation. S.T.T.D. supervised the sampling, isolation, AST procedures and revised the manuscript. A.D., Y.M.G.H., J.E.O., and F.S. provided critical review and editing of the manuscript. All authors have read and agreed to the published version of the manuscript.

Funding: This research study was funded by the Danish International Development Assistance (DANIDA) to the project "Health and Antibiotics in Vietnamese Pig Production" (grant DFC file number 17-M06-KU).

Institutional Review Board Statement: The study was conducted according to the guidelines of the Declaration of Helsinki, and approved by the Ethical Committee of the National Institute of Nutrition in Hanoi (Certificate No. 04/VDD-QLKH).

Informed Consent Statement: Oral informed consent was obtained from all pig farm workers involved in the study.

Data Availability Statement: All data supporting this study are available in the supplementary materials. Moreover, the genome sequence reads have been deposited to the European Nucleotide Archive and publicly available with the accession number PRJEB37980.

Acknowledgments: We would like to thank the staff of the Sub-Department of Animal Health in Bac Ninh Province for their support in the sample collection. We also thank the staff at the Department of Veterinary and Animal Sciences, University of Copenhagen, for their laboratory technical assistance.

Conflicts of Interest: The authors declare there are no conflicting interests that could influence the publication of this study.

\section{References}

1. WHO. Global Action Plan on Antimicrobial Resistance; WHO: Geneva, Switzerland, 2017; pp. 1-28.

2. Chereau, F.; Opatowski, L.; Tourdjman, M.; Vong, S. Risk Assessment for Antibiotic Resistance in South East Asia. BMJ 2017, 358. Available online: https:/ / www.ncbi.nlm.nih.gov/pmc/articles/PMC5598255/ (accessed on 5 September 2017).

3. Vounba, P.; Arsenault, J.; Bada-Alambédji, R.; Fairbrother, J.M. Pathogenic potential and the role of clones and plasmids in beta-lactamase-producing E. coli from chicken faeces in Vietnam. BMC Vet. Res. 2019, 15, 106. [CrossRef]

4. Mburu, J.; Egelyng, H.; Ackello-ogutu, C. Household Pork Consumption Behaviour in Vietnam: Implications for pro-Smallholder Pig Value Chain Upgrading. In Proceedings of the Tropentag 2015, Berlin, Germany, 16-18 September 2015.

5. Lucila, M.; Lapar, A. The REVALTER Project "Multi-Scale Assessment of Livestock Development Pathways in Vietnam"; Review of the Pig Sector in Vietnam; ILRI: Nairobi, Kenya, 2014.

6. Carrique-Mas, J.J.; Choisy, M.; Van Cuong, N.; Thwaites, G.; Baker, S. An estimation of total antimicrobial usage in humans and animals in Vietnam. Antimicrob. Resist. Infect. Control 2020, 9, 16. [CrossRef]

7. Van Cuong, N.; Nhung, N.T.; Nghia, N.H.; Mai Hoa, N.T.; Trung, N.V.; Thwaites, G.; Carrique-Mas, J. Antimicrobial Consumption in Medicated Feeds in Vietnamese Pig and Poultry Production. Ecohealth 2016, 13, 490-498. [CrossRef] [PubMed]

8. WHO. List of Critically Important Antimicrobials for Human Medicine; Who: Geneva, Switzerland, 2017 ; ISBN 9789241504485.

9. Nguyen, N.T.; Nguyen, H.M.; Nguyen, C.V.; Nguyen, T.V.; Nguyen, M.T.; Thai, H.Q.; Ho, M.H.; Thwaites, G.; Ngo, H.T.; Baker, S.; et al. Use of Colistin and Other Critical Antimicrobials on Pig and Chicken Farms in Southern Vietnam and Its Association with Resistance in Commensal Escherichia coli Bacteria. Appl. Environ. Microbiol. 2016, 82, 3727-3735. [CrossRef] [PubMed] 
10. Dang, S.T.T.; Bortolaia, V.; Tran, N.T.; Le, H.Q.; Dalsgaard, A. Cephalosporin-resistant Escherichia coli isolated from farm workers and pigs in northern Vietnam. Trop. Med. Int. Health 2018, 23, 415-424. [CrossRef]

11. Nguyen, V.T.; Carrique-Mas, J.J.; Ngo, T.H.; Ho, H.M.; Ha, T.T.; Campbell, J.I.; Nguyen, T.N.; Hoang, N.N.; Pham, V.M.; Wagenaar, J.A.; et al. Prevalence and risk factors for carriage of antimicrobial-resistant Escherichia coli on household and small-scale chicken farms in the Mekong Delta of Vietnam. J. Antimicrob. Chemother. 2015, 70, 2144-2152. [PubMed]

12. Mitchell, M.E.; Alders, R.; Unger, F.; Nguyen-Viet, H.; Le TT, H.; Toribio, J.A. The challenges of investigating antimicrobial resistance in Vietnam — what benefits does a One Health approach offer the animal and human health sectors? BMC Public Health 2020, 20,1-12. Available online: https:/ / bmcpublichealth.biomedcentral.com/articles/10.1186/s12889-020-8319-3 (accessed on 24 September 2021). [CrossRef] [PubMed]

13. Ueda, S.; Ngan, B.T.K.; Huong, B.T.M.; Hirai, I.; Tuyen, L.D.; Yamamoto, Y. Limited Transmission of blaCTX-M-9-Type-Positive Escherichia coli between Humans and Poultry in Vietnam. Antimicrob. Agents Chemother. 2015, 59, 3574-3577. [CrossRef]

14. Börjesson, S.; Ny, S.; Egervärn, M.; Bergström, J.; Rosengren, Å.; Englund, S.; Löfmark, S.; Byfors, S. Limited Dissemination of Extended-Spectrum $\beta$-Lactamase- and Plasmid-Encoded AmpC-Producing Escherichia coli from Food and Farm Animals, Sweden. Emerg. Infect. Dis. 2016, 22, 634-640. [CrossRef]

15. Dorado-García, A.; Smid, J.H.; van Pelt, W.; Bonten, M.J.M.; Fluit, A.C.; van den Bunt, G.; Wagenaar, J.A.; Hordijk, J.; Dierikx, C.M.; Veldman, K.T.; et al. Molecular relatedness of ESBL/AmpC-producing Escherichia coli from humans, animals, food and the environment: A pooled analysis. J. Antimicrob. Chemother. 2018, 73, 339-347. [CrossRef] [PubMed]

16. Nguyen, V.T.; Jamrozy, D.; Matamoros, S.; Carrique-Mas, J.J.; Ho, H.M.; Thai, Q.H.; Nguyen, T.N.M.; Wagenaar, J.A.; Thwaites, G.; Parkhill, J.; et al. Limited Contribution of Non-Intensive Chicken Farming to Esbl-Producing Escherichia Coli Colonization in Humans in Vietnam: An Epidemiological and Genomic Analysis. J. Antimicrob. Chemother. 2019, 74, 561-570. [CrossRef] [PubMed]

17. Ludden, C.; Raven, K.E.; Jamrozy, D.; Gouliouris, T.; Blane, B.; Coll, F.; de Goffau, M.; Naydenova, P.; Horner, C.; Hernandez-Garcia, J.; et al. One Health Genomic Surveillance of Escherichia coli Demonstrates Distinct Lineages and Mobile Genetic Elements in Isolates from Humans versus Livestock. MBio 2019, 10, e02693-18. [CrossRef] [PubMed]

18. Johnson, J.R.; Murray, A.C.; Gajewski, A.; Sullivan, M.; Snippes, P.; Kuskowski, M.A.; Smith, K.E. Isolation and Molecular Characterization of Nalidixic Acid-Resistant Extraintestinal Pathogenic Escherichia coli from Retail Chicken Products. Antimicrob. Agents Chemother. 2003, 47, 2161-2168. [CrossRef]

19. Spurbeck, R.R.; Dinh, P.C.; Walk, S.T.; Stapleton, A.E.; Hooton, T.M.; Nolan, L.K.; Kim, K.S.; Johnson, J.R.; Mobley, H.L.T. Escherichia coli Isolates That Carry vat, fyuA, chuA, and yfcV Efficiently Colonize the Urinary Tract. Infect. Immun. 2012, 80, 4115-4122. [CrossRef]

20. Day, M.J.; Rodríguez, I.; van Essen-Zandbergen, A.; Dierikx, C.; Kadlec, K.; Schink, A.-K.; Wu, G.; Chattaway, M.A.; DoNascimento, V.; Wain, J.; et al. Diversity of STs, plasmids and ESBL genes among Escherichia coli from humans, animals and food in Germany, the Netherlands and the UK. J. Antimicrob. Chemother. 2016, 71, 1178-1182. [CrossRef]

21. Flament-Simon, S.-C.; Nicolas-Chanoine, M.-H.; García, V.; Duprilot, M.; Mayer, N.; Alonso, M.P.; García-Meniño, I.; Blanco, J.E.; Blanco, M.; Blanco, J. Clonal Structure, Virulence Factor-encoding Genes and Antibiotic Resistance of Escherichia coli, Causing Urinary Tract Infections and Other Extraintestinal Infections in Humans in Spain and France during 2016. Antibiotics 2020, 9, 161. [CrossRef]

22. Chattaway, M.A.; Jenkins, C.; Ciesielczuk, H.; Day, M.; DoNascimento, V.; Day, M.; Rodríguez, I.; van Essen-Zandbergen, A.; Schink, A.-K.; Wu, G.; et al. Evidence of Evolving Extraintestinal Enteroaggregative Escherichia coli ST38 Clone. Emerg. Infect. Dis. 2014, 20, 1935-1937. [CrossRef]

23. Ahmed, S.; Olsen, J.E.; Herrero-Fresno, A. The genetic diversity of commensal Escherichia coli strains isolated from nonantimicrobial treated pigs varies according to age group. PLOS ONE 2017, 12, e0178623. [CrossRef]

24. Bui, T.K.N.; Bui, T.M.H.; Ueda, S.; Le, D.T.; Yamamoto, Y.; Hirai, I. Potential transmission opportunity of CTX-M-producing Escherichia coli on a large-scale chicken farm in Vietnam. J. Glob. Antimicrob. Resist. 2018, 13, 1-6. [CrossRef] [PubMed]

25. Hoang, T.a.V.; Nguyen, T.N.H.; Ueda, S.; Le, Q.P.; Tran, T.T.N.; Nguyen, T.N.D.; Dao, T.V.K.; Tran, M.T.; Le, T.T.T.; Le, T.L.; et al. Common findings of bla CTX-M-55-encoding 104-139 kbp plasmids harbored by extended-spectrum $\beta$-lactamase-producing Escherichia coli in pork meat, wholesale market workers, and patients with urinary tract infection in Vietnam. Curr. Microbiol. 2017, 74, 203-211. [CrossRef]

26. Kawahara, R.; Khong, D.T.; Le, H.V.; Phan, Q.N.; Nguyen, T.N.; Yamaguchi, T.; Kumeda, Y.; Yamamoto, Y. Prevalence Of mcr-1 Among Cefotaxime-Resistant Commensal Escherichia coli In Residents Of Vietnam. Infect. Drug Resist. 2019, 12, 3317-3325. [CrossRef]

27. Nakayama, T.; Ueda, S.; Huong, B.T.M.; Tuyen, L.D.; Komalamisra, C.; Kusolsuk, T.; Hirai, I.; Yamamoto, Y. Wide dissemination of extended-spectrum $\beta$-lactamase-producing Escherichia coli in community residents in the Indochinese peninsula. Infect. Drug Resist. 2015, 8, 1-5. [CrossRef]

28. Nguyen, D.P.; Nguyen, T.A.D.; Le, T.H.; Tran, N.M.D.; Ngo, T.P.; Dang, V.C.; Kawai, T.; Kanki, M.; Kawahara, R.; Jinnai, M.; et al. Dissemination of Extended-Spectrum $\beta$-Lactamase-and AmpC $\beta$-Lactamase-Producing Escherichia coli within the Food Distribution System of Ho Chi Minh City, Vietnam. BioMed Res. Int. 2016. [CrossRef] 
29. Hinenoya, A.; Tran, S.T.T.; Nguyen, N.T.; Nguyen, H.C.; Nguyen, D.D.L.; Hoang, P.H.; Awasthi, S.P.; Hassan, J.; Sumimura, Y.; Yamamoto, Y.; et al. Isolation and molecular characterization of extended-spectrum $\beta$-lactamase producing Escherichia coli from industrial food animals in Mekong Delta, Vietnam. Jpn. J. Vet. Res. 2018, 66, 1-12.

30. LaPlante, K.; Cusumano, J.; Tillotson, G. Colistin for the treatment of multidrug-resistant infections. Lancet Infect. Dis. 2018, 18, 1174-1175. [CrossRef]

31. Teramae, M.; Osawa, K.; Shigemura, K.; Kitagawa, K.; Shirakawa, T.; Fujisawa, M.; Miyara, T. Prevalence of Quinolone Resistance of Extended-Spectrum $\beta$-Lactamase-Producing Escherichia coli with ST131-fimH30 in a City Hospital in Hyogo, Japan. Int. J. Mol. Sci. 2019, 20, 5612. [CrossRef] [PubMed]

32. Peirano, G.; Pitout, J.D.D. Fluoroquinolone-Resistant Escherichia coli Sequence Type 131 Isolates Causing Bloodstream Infections in a Canadian Region with a Centralized Laboratory System: Rapid Emergence of the H30-Rx Sublineage. Antimicrob. Agents Chemother. 2014, 58, 2699-2703. [CrossRef]

33. Matsumura, Y.; Johnson, J.R.; Yamamoto, M.; Nagao, M.; Tanaka, M.; Takakura, S.; Ichiyama, S.; Kyoto-Shiga Clinical Microbiology Study Group. Kyoto-Shiga Clinical Microbiology Study Group CTX-M-27- and CTX-M-14-producing, ciprofloxacin-resistant Escherichia coli of the H30 subclonal group within ST131 drive a Japanese regional ESBL epidemic. J. Antimicrob. Chemother. 2015, 70, 1639-1649.

34. Zurfluh, K.; Abgottspon, H.; Hächler, H.; Nüesch-Inderbinen, M.; Stephan, R. Quinolone Resistance Mechanisms among Extended-Spectrum Beta-Lactamase (ESBL) Producing Escherichia coli Isolated from Rivers and Lakes in Switzerland. PLoS ONE 2014, 9, e95864.

35. Nhung, N.T.; Cuong, N.V.; Thwaites, G.; Carrique-Mas, J. Antimicrobial Usage and Antimicrobial Resistance in Animal Production in Southeast Asia: A Review. Antibiotics 2016, 5, 37. [CrossRef]

36. Mathers, A.J.; Peirano, G.; Pitout, J.D.D. The Role of Epidemic Resistance Plasmids and International High-Risk Clones in the Spread of Multidrug-Resistant Enterobacteriaceae. Clin. Microbiol. Rev. 2015, 28, 565-591. [CrossRef]

37. Yamamoto, Y.; Kawahara, R.; Fujiya, Y.; Sasaki, T.; Hirai, I.; Khong, D.T.; Nguyen, T.N.; Nguyen, B.X. Wide dissemination of colistin-resistant Escherichia coli with the mobile resistance gene $\mathrm{mcr}$ in healthy residents in Vietnam. J. Antimicrob. Chemother. 2019, 74, 523-524. [CrossRef]

38. Bui, T.M.H.; Hirai, I.; Ueda, S.; Bui, T.K.N.; Hamamoto, K.; Toyosato, T.; Le, D.T.; Yamamoto, Y. Carriage of Escherichia coli Producing CTX-M-Type Extended-Spectrum $\beta$-Lactamase in Healthy Vietnamese Individuals. Antimicrob. Agents Chemother. 2015, 59, 6611-6614. [CrossRef]

39. Zając, M.; Sztromwasser, P.; Bortolaia, V.; Leekitcharoenphon, P.; Cavaco, L.M.; Ziętek-Barszcz, A.; Hendriksen, R.S.; Wasyl, D. Occurrence and Characterization of mcr-1-Positive Escherichia coli Isolated From Food-Producing Animals in Poland, 2011-2016. Front. Microbiol. 2019, 10, 1753. [CrossRef]

40. Magiorakos, A.P.; Srinivasan, A.; Carey, R.B.; Carmeli, Y.; Falagas, M.E.; Giske, C.G.; Harbarth, S.; Hindler, J.F.; Kahlmeter, G.; Olsson-Liljequist, B.; et al. Multidrug-resistant, Extensively Drug-Resistant and Pandrug-Resistant Bacteria: An International Expert Proposal for Interim Standard Definitions for Acquired Resistance-PubMed. Clin. Microbiol. Infect 2012, 18, 268-281. [CrossRef]

41. Yamasaki, S.; Le, T.D.; Vien, M.Q.; Van Dang, C.; Yamamoto, Y. Prevalence of extended-spectrum $\beta$-lactamase-producing Escherichia coli and residual antimicrobials in the environment in Vietnam. Anim. Health Res. Rev. 2017, 18, 128-135. [CrossRef] [PubMed]

42. Vounba, P.; Rhouma, M.; Arsenault, J.; Bada Alambédji, R.; Fravalo, P.; Fairbrother, J.M. Prevalence of colistin resistance and mcr-1/mcr-2 genes in extended-spectrum $\beta$-lactamase / AmpC-producing Escherichia coli isolated from chickens in Canada, Senegal and Vietnam. J. Glob. Antimicrob. Resist. 2019, 19, 222-227. [CrossRef]

43. Coyne, L.; Arief, R.; Benigno, C.; Giang, V.N.; Huong, L.Q.; Jeamsripong, S.; Kalpravidh, W.; McGrane, J.; Padungtod, P.; Patrick, I.; et al. Characterizing Antimicrobial Use in the Livestock Sector in Three South East Asian Countries (Indonesia, Thailand, and Vietnam). Antibiotics 2019, 8, 33. [CrossRef]

44. Nguyen, K.V.; Thi Do, N.T.; Chandna, A.; Nguyen, T.V.; Pham, C.V.; Doan, P.M.; Nguyen, A.Q.; Thi Nguyen, C.K.; Larsson, M.; Escalante, S.; et al. Antibiotic use and resistance in emerging economies: A situation analysis for Viet Nam. BMC Public Health 2013, 13, 1158. [CrossRef]

45. The List of Veterinary Drugs has been Licensed for Circulation in Vietnam until March 2019 (Vietnamese). Available online: http:/ / cucthuy.gov.vn/Pages/danh-muc-thuoc-thu-y-da-duoc-cap-giay-chung-nhan-luu-hanh-tai-viet-nam-.aspx (accessed on 3 May 2020).

46. Yang, S.; Deng, W.; Liu, S.; Yu, X.; RazaMustafa, G.; Chen, S.; He, L.; Ao, X.; Yang, Y. Presence of Heavy Metal Resistance Genes in Escherichia coli and Salmonella, and Analysis of Resistance Gene Structure in E. coli E308. J. Glob. Antimicrob. Resist. 2020, 21, 420-426. [CrossRef]

47. Comparative analysis of the susceptibility to biocides and heavy metals of extended-spectrum $\beta$-lactamase-producing Escherichia coli isolates of human and avian origin, Germany. Diagn. Microbiol. Infect. Dis. 2017, 88, 88-92. [CrossRef]

48. Yu, Z.; Gunn, L.; Wall, P.; Fanning, S. Antimicrobial resistance and its association with tolerance to heavy metals in agriculture production. Food Microbiol. 2017, 64, 23-32. [CrossRef]

49. Yang, Q.E.; Agouri, S.R.; Tyrrell, J.M.; Walsh, T.R. Heavy Metal Resistance Genes Are Associated with blaNDM-1- and blaCTX-M15-Carrying Enterobacteriaceae. Antimicrob. Agents Chemother. 2018, 62, e02642-17. [CrossRef] 
50. EUCAST: Clinical Breakpoints and Dosing of Antibiotics. Available online: https:/ / eucast.org/clinical_breakpoints/ (accessed on 12 April 2020).

51. Farmer, J.J.; Farmer, M.K.; Holmes, B. The Enterobacteriaceae: General Characteristics. In Topley E Wilson's Microbiology and Microbial Infections; American Cancer Society: Chichester, UK, 2010; ISBN 978-0-470-68861-8.

52. CLSI M100 I Performance Standards for Antimicrobial Susceptibility Testing, 28th Edition. Available online: https://clsi.org/ standards/products/microbiology/documents/m100/ (accessed on 12 April 2020).

53. Naseer, F.; Iqbal, R.; Ikram, N.; Shoaib, M.; asad, M.J.; Mehmood, R.T.; Niazi, A.; Niazi, A.; Ishfaq, B. Phenotypic cofirmatory disc diffusion test (PCDDT), double disc synergy test (DDST), E-test OS diagnostic tool for detection of extended spectrum beta lactamase (ESBL) producing Uropathogens. Appl. Biotechnol. Bioeng. Appl. Biotechnol 2017, 3, 344-349. [CrossRef]

54. Bankevich, A.; Nurk, S.; Antipov, D.; Gurevich, A.A.; Dvorkin, M.; Kulikov, A.S.; Lesin, V.M.; Nikolenko, S.I.; Pham, S.; Prjibelski, A.D.; et al. SPAdes: A new genome assembly algorithm and its applications to single-cell sequencing. J. Comput. Biol. J. Comput. Mol. Cell Biol. 2012, 19, 455-477. [CrossRef]

55. Gurevich, A.; Saveliev, V.; Vyahhi, N.; Tesler, G. QUAST: Quality assessment tool for genome assemblies. Bioinform. Oxf. Engl. 2013, 29, 1072-1075. [CrossRef] [PubMed]

56. Malberg Tetzschner, A.M.; Johnson, J.R.; Johnston, B.D.; Lund, O.; Scheutz, F. In Silico Genotyping of Escherichia coli Isolates for Extraintestinal Virulence Genes by Use of Whole-Genome Sequencing Data. J. Clin. Microbiol. 2020, 58, e01269-20. Available online: https: / /journals.asm.org/doi/10.1128/JCM.01269-20 (accessed on 20 August 2021). [CrossRef]

57. RASTtk: A Modular and Extensible Implementation of the RAST Algorithm for Building Custom Annotation Pipelines and Annotating Batches of Genomes. Sci. Rep. 2015, 5, 1-6. Available online: https://www.nature.com/articles/srep08365 (accessed on 3 May 2020).

58. Kaas, R.S.; Leekitcharoenphon, P.; Aarestrup, F.M.; Lund, O. Solving the Problem of Comparing Whole Bacterial Genomes across Different Sequencing Platforms. PLoS ONE 2014, 9, e104984. [CrossRef]

59. Interactive Tree of Life (iTOL) v3: An Online Tool for the Display and Annotation of Phylogenetic and other Trees. Available online: https:/ / www.ncbi.nlm.nih.gov/pmc/articles/PMC4987883/ (accessed on 12 April 2020). 